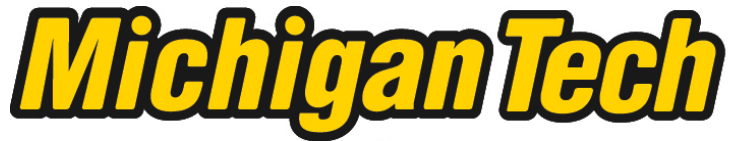 \\ Michigan Technological University Create the Future Digital Commons @ Michigan Tech
}

Dissertations, Master's Theses and Master's Reports - Open

Dissertations, Master's Theses and Master's

Reports

2011

Tracking a non-cooperative target using a Doppler radar wireless sensor network

Michael K. Blaser

Michigan Technological University

Follow this and additional works at: https://digitalcommons.mtu.edu/etds

Part of the Electrical and Computer Engineering Commons

Copyright 2011 Michael K. Blaser

\section{Recommended Citation}

Blaser, Michael K., "Tracking a non-cooperative target using a Doppler radar wireless sensor network", Master's report, Michigan Technological University, 2011.

https://doi.org/10.37099/mtu.dc.etds/573

Follow this and additional works at: https://digitalcommons.mtu.edu/etds

Part of the Electrical and Computer Engineering Commons 


\title{
Tracking a Non-Cooperative Target Using a Doppler Radar Wireless Sensor Network
}

\author{
By \\ Michael K. Blaser \\ A REPORT \\ Submitted in partial fulfillment of the requirements for the degree of \\ MASTER OF SCIENCE \\ Electrical Engineering \\ MICHIGAN TECHNOLOGICAL UNIVERSITY \\ 2011
}

(C)2011 Michael K. Blaser 
This report, "Tracking a Non-Cooperative Target Using a Doppler Radar Wireless Sensor Network," is hereby approved in partial fulfillment of the requirements for the Degree of MASTER OF SCIENCE IN ELECTRICAL ENGINEERING.

Department of Electrical and Computer Engineering

Signatures:

\begin{tabular}{cl} 
Report Advisor & \\
Report Co-Advisor & \\
Department Chair Shiyan Hu & \\
& \\
& \\
& \\
\hline
\end{tabular}




\section{Contents}

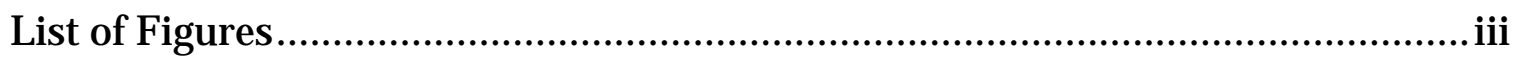

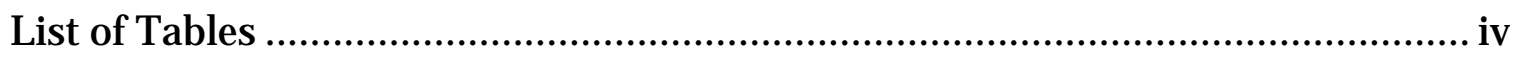

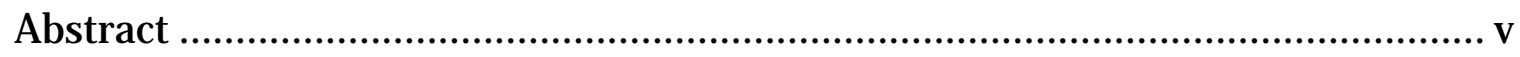

Chapter 1 Introduction.................................................................................... 1

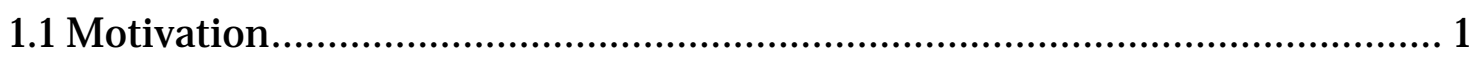

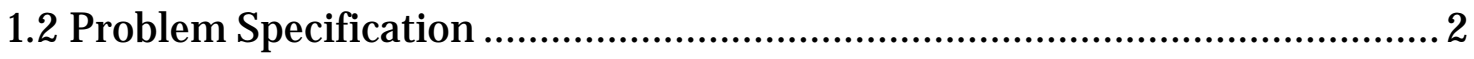

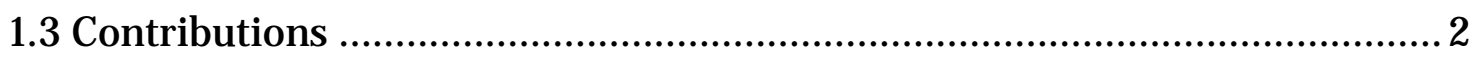

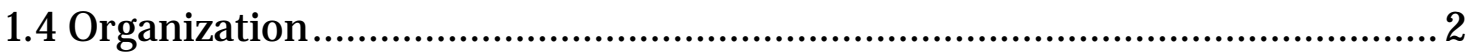

Chapter 2 Related Work............................................................................. 3

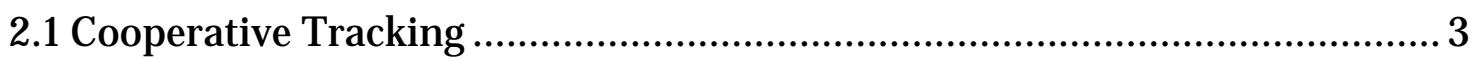

2.2 Non-Cooperative Tracking .......................................................................... 3

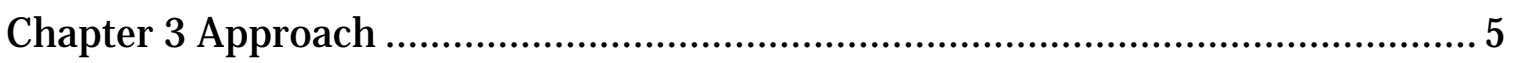

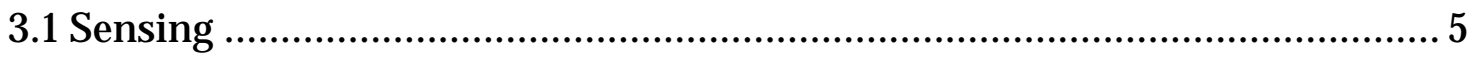

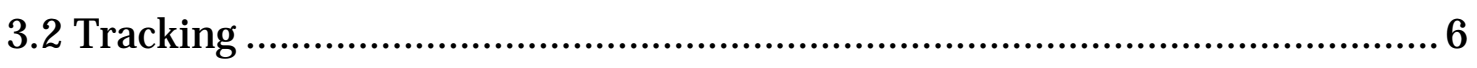

3.2.1 Extended Kalman Filter................................................................... 6

Chapter 4 Implementation................................................................................ 10

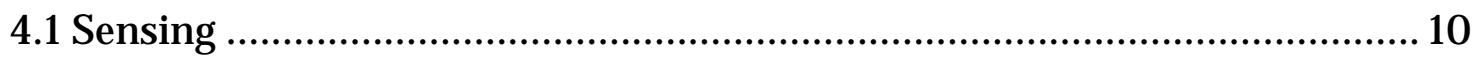

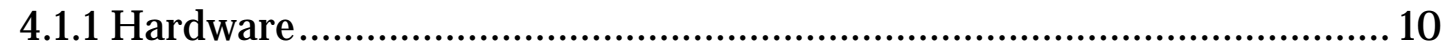

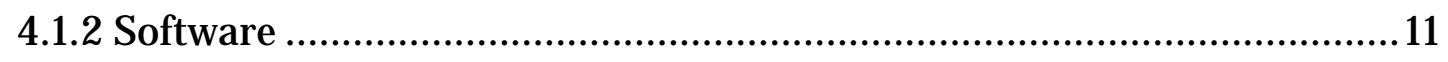

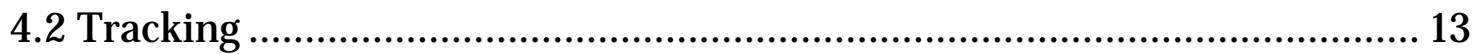




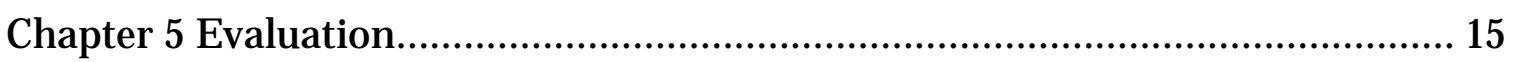

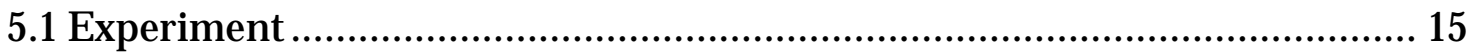

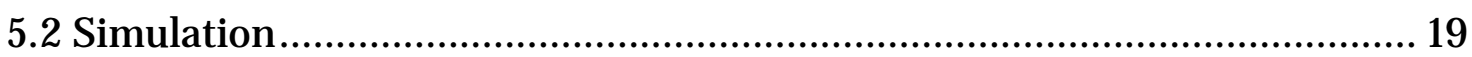

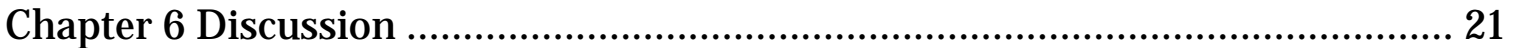

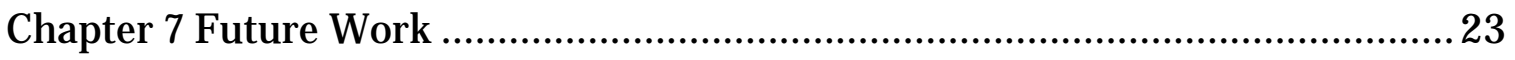

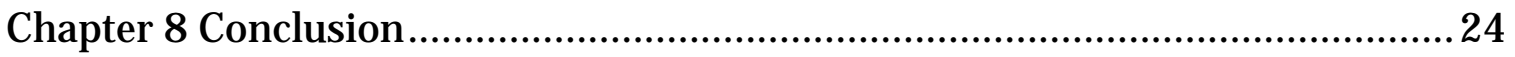

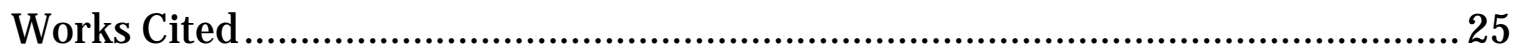

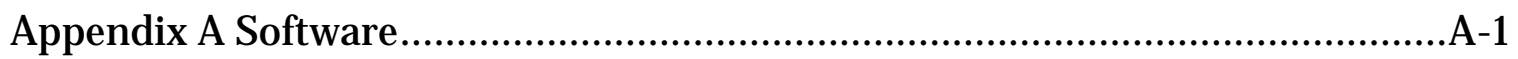

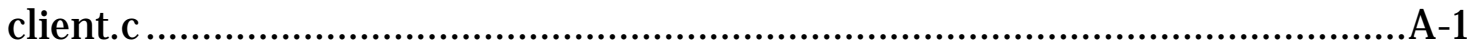

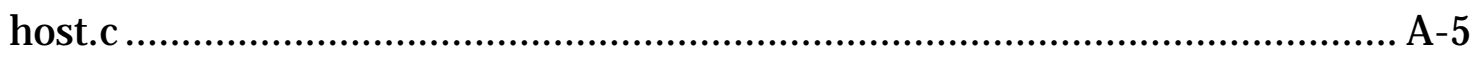

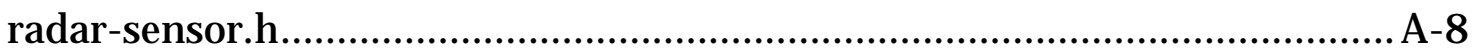

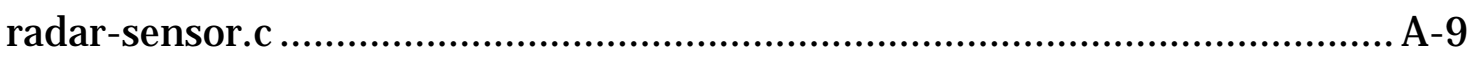

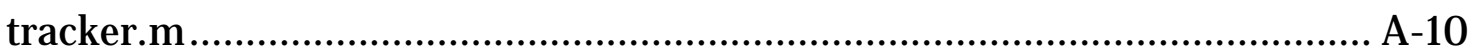

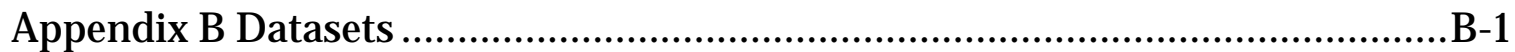

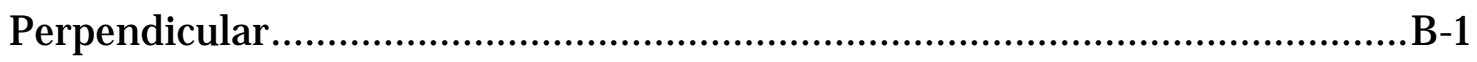

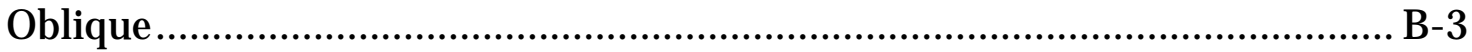

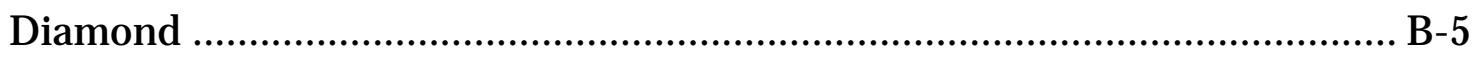




\section{List of Figures}

Figure 3.1: General layout of sensor node radars in reference to the target........... 5

Figure 3.2: Shows the radar and target layout. ................................................... 8

Figure 4.1: System Block Diagram of the tracking WSN....................................... 10

Figure 4.2: The two WSN hardware Platforms used.............................................11

Figure 4.3: Block Diagram of client.c code flow. .............................................. 12

Figure 4.4: Block Diagram of host.c code flow. .................................................... 13

Figure 4.5: Block diagram of tracker.m program flow. ......................................... 14

Figure 5.1: Diagram of experiment and simulation layout.................................... 15

Figure 5.2: Experimental results for the perpendicular test pattern. ................... 16

Figure 5.3: Experimental results for the oblique test pattern.................................17

Figure 5.4: Experimental results for the diamond test pattern. ........................... 18

Figure 5.5: Simulation results of perpendicular test pattern................................ 19

Figure 5.6: Simulation results of oblique test pattern...........................................20 


\section{List of Tables}

Table 3.1: Extended Kalman Filter Algorithm................................................ 7

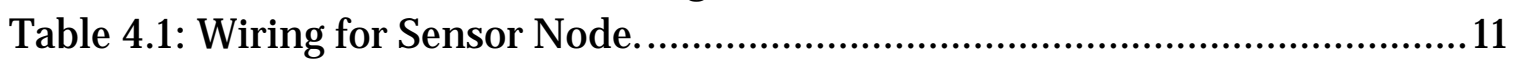

Table 6.1: Statistical summary for experimental perpendicular test pattern. ...... 21

Table 6.2: Statistical summary for experimental oblique test pattern. ................ 21 


\section{Abstract}

Tracking or target localization is used in a wide range of important tasks from knowing when your flight will arrive to ensuring your mail is received on time. Tracking provides the location of resources enabling solutions to complex logistical problems. Wireless Sensor Networks (WSN) create new opportunities when applied to tracking, such as more flexible deployment and real-time information. When radar is used as the sensing element in a tracking WSN better results can be obtained; because radar has a comparatively larger range both in distance and angle to other sensors commonly used in WSNs. This allows for less nodes deployed covering larger areas, saving money.

In this report I implement a tracking WSN platform similar to what was developed by Lim, Wang, and Terzis [1]. This consists of several sensor nodes each with a radar, a sink node connected to a host PC, and a Matlab@ program to fuse sensor data. I have re-implemented their experiment with my WSN platform for tracking a non-cooperative target to verify their results and also run simulations to compare. The results of these tests are discussed and some future improvements are proposed. 


\section{Chapter 1 Introduction}

\subsection{Motivation}

Tracking or target localization is used in a wide range of important tasks from knowing when your flight will arrive to ensuring your mail is received on time. Tracking provides the location of resources enabling solutions to complex logistical problems.

Wireless Sensor Networks (WSN) allow for new opportunities when applied to tracking. More flexible deployment of a tracking network can be achieved since each sensor node is wireless. Together the WSN can provide realtime information on what it is tracking.

By using radar as the sensing element in the tracking WSN better results can be obtained. Radar has a comparatively larger range both in distance and angle to other sensors commonly used in WSNs. This allows for less nodes deployed covering larger areas, saving money. This introduces many new possible applications such as intruder detection or elderly persons monitoring.

Intruder detection when applied to WSNs is the tracking of an invader into the networks domain [2]. This has been a major topic of interest for the military to deploy on battle fields but could also be applied to simple building security. By implementing intruder detection with a WSN it greatly automates the task and allows for real-time information.

Another application of tracking in WSNs is to monitor the elderly [3]. The elderly, in partially assisted living, wish to keep their independence but want the security of knowing someone can help them. By using a WSN to track a patient it is possible to determine if they have fallen or are in need of assistance, and allows for up to the date information on their location. 


\subsection{Problem Specification}

Tracking in WSNs can be broken into three sub problems which apply to all WSNs; sensing, networking, and data fusion. Quality data must be obtained from each sensor node to provide accurate results. Each node must be networked together so data and commands can be communicated to and from each node. All the data from the WSN should be somehow combined to provide better information to our tracking goal.

\subsection{Contributions}

In this report a tracking WSN platform similar to what was developed by Lim, Wang, and Terzis is implemented [1]. Our platform consists of several sensor nodes each with a radar, a sink node connected to a host PC, and a Matlab (C) program to fuse sensor data. We have re-implemented their experiment with our WSN platform for tracking a non-cooperative target to verify their results and simulations to compare. The results of these tests are discussed and some future improvements are proposed.

\subsection{Organization}

The remainder of this report is organized as follows:

- Chapter 1 - Introduction

- Chapter 2 - This section explores previous work done on tracking with WSNs for both cooperative and non-cooperative methods.

- Chapter 3 - This section gives a general approach of the techniques and methods I use to create a non-cooperative tracking WSN.

- Chapter 4 - This section describes in detail the hardware and software used to implement the WSN.

- Chapter 5 - This section describes the tests and simulations performed to evaluate the WSN and show their results.

- Chapter 6 - This section analyzes the results from the tests, discusses these results, and shows some of the limitations of the WSN.

- Chapter 7 - This section looks at several methods that could be used to reduce the limitations found from the experiments for future work.

- Chapter 8 - Conclusion 


\section{Chapter 2 Related Work}

Tracking using a WSN is by no means a new concept and some of the first to explore it were Bahl and Padmanabhan in the year 2000 [4]. Their WSN used received signal strength (RSS) from a sensor on the target and multiple distributed sensors to track people through a building. Since the target is an active element in the tracking algorithm this method is considered cooperative tracking. The majority of research on tracking with WSNs has been done with cooperative tracking.

\subsection{Cooperative Tracking}

The breadth of cooperative tracking research is quite large so the following are just so distinct examples of the area. In "Simultaneous Localization and Mobile Robot Navigation in a Hybrid Sensor Network" the authors explore using a mobile robot to simultaneously localize both the robot and the WSN without an explicit distance sensor [5]. They use radio messages which have some range in combination with the moving robots odometry and by moving in and out of the range of the sensors allows for both the robot and the sensors to localize. The well known Cricket WSN platform developed by Priyantha, Chakraborty, and Balakrishnan used both radio and sound wave in a time difference of arrival method to localize nodes [6]. Lastly "Tracking Mobile Nodes Using RF Doppler Shifts" measures the Doppler shift of a node's radio beacon to other stationary nodes [7]. All of these examples are limited to have a node on the tracking target and this is impractical for some applications such as intruder detection.

\subsection{Non-Cooperative Tracking}

Non-cooperative tracking attempts to solve the tracking problem without the target actively participating. This makes tracking problem more difficult since it puts more importance on the sensing element. Therefore the different 
approaches to non-cooperative tracking are based around different sensing technologies.

Ultrasonic sensors provide accurate, semi-directional information. Nishida, et al. use ultrasonic sensors embedded into the ceiling of a roof to localize humans based on head positions [8]. This allows for the minimally invasive tracking people down to $5 \mathrm{~cm}$ in a building but is limited to only people. $\mathrm{Li}$, et al. attempts to track generic moving objects with sensor "poles" which have multiple ultrasonic sensors at different angles on them [9]. This allows for more flexible tracking but requires poles in a grid spaced $2.5 \mathrm{~m}$ apart.

Camera based WSNs allow for the greatest flexibility with large amounts of possible tracking information but are the processing intensive. SanchezMatamoros, Dio, and Ollero used vision-based WSN to detect, localize, and track objects [10]. Their solution focused on processing the images and reducing the needed network bandwidth. The energy need to process images is prohibitive to the lifetime of the WSN.

While ultrasonic and camera based WSN are more common there are still many other techniques. He, et al. implemented a large scale WSN with 200 nodes to track vehicles over a $300 \mathrm{~m}$ and $200 \mathrm{~m}$ stretch of road called VigilNet [11]. The sensing mechanism combined multiple low fidelity, low cost sensors; acoustic, photo, temperature, and passive infrared. This allows for a binary detection scheme which provides low resolution but great scalability as 200 nodes covered a $5000 \mathrm{~m}^{2}$ area. Mao, et al. uses a creative solution involving room light fixture and with groups of extremely low cost light sensors to track a person moving down a hall [12]. While the sensors were very low cost they were still able to localize a target to within $20 \mathrm{~cm}$ but needed 40 sensors to cover one $12.5 \mathrm{~m}$ hallway. 


\section{Chapter 3 Approach}

We divide the non-cooperative tracking problem up into two part, sensing and tracking. The distributed WSN nodes collect information and then send it to a sink node connected to a host PC as seen in Figure 3.1. Software on the Host PC fuses all of the sensor information, tracks the target, and provides real-time output.

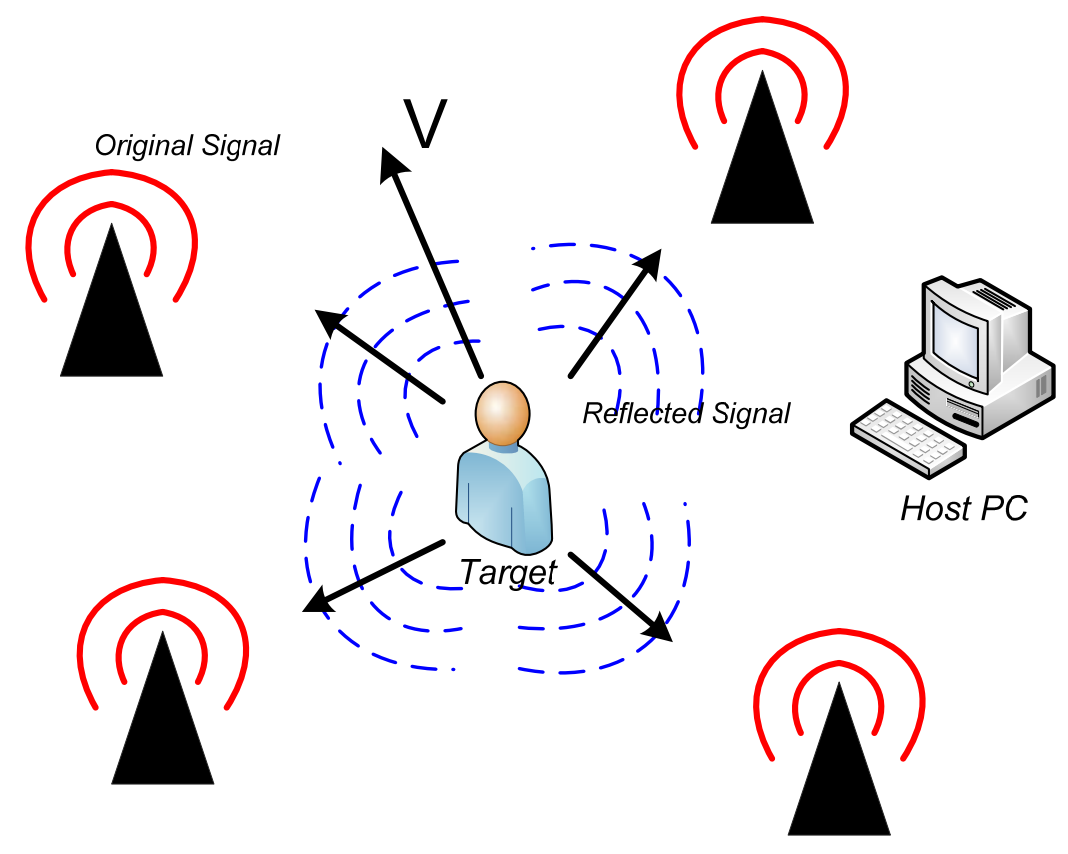

Figure 3.1: General layout of sensor node radars in reference to the target.

\subsection{Sensing}

Each WSN sensor node has a BumbleBee radar to collect Doppler frequency information from the target movement. This information is then processed with 
autocorrelation to find the target's Doppler frequency using Equation (3.1) and Equation (3.2)

$$
\begin{gathered}
R_{n}(1)=\sum_{n} x_{n} x_{n-1}^{*} \\
\angle R_{n}(1)=\tan ^{-1} \frac{i m\left\{R_{n}(1)\right\}}{r e\left\{R_{n}(1)\right\}}
\end{gathered}
$$

Autocorrelation will give the average frequency from the Fourier Transform of the Doppler information, and because there is only one target within the WSN field this should be the Doppler shift frequency and by applying Equation (3.3) we can calculate the velocity of the target.

$$
V_{d}=\frac{f_{d} \lambda}{2}
$$

After the velocity is calculated it is sent off the sink node over the WSN as a UDP packet. The sink node collects all UDP packets from the sensor nodes.

\subsection{Tracking}

The sink node receives all incoming UDP packets from the sensors taking each and transmits them to the host PC as a string message. The host PC runs Matlab (C) which reads in the messages, feeds them into an Extended Kalman Filter (EKF), and outputs the current estimation of the EKF.

\subsubsection{Extended Kalman Filter}

The EKF is used over a traditional Kalman Filter because the observation model is non-linear; although the state transition model is linear. Shown in Table 3.1 is the EKF algorithm used in our research. 
Table 3.1: Extended Kalman Filter Algorithm.

\begin{tabular}{|l} 
Priori \\
$\hat{x}_{k}^{-}=A \hat{x}_{k-1}$ \\
$P_{k}^{-}=A P_{k-1}^{-} A^{T}+Q$ \\
Posteriori \\
$K_{k}=\frac{P_{k}^{-} J_{k}^{T}}{J_{k} P_{k}^{-} J_{k}^{T}+R}$ \\
$\hat{x}_{k}=\hat{x}_{k}^{-}+K_{k}\left(z_{k}-F\left(\hat{x}_{k}^{-}\right)\right)$ \\
$P_{k}=\left(\mathbb{I}-K_{k} J_{k}\right) P_{k}^{-}$
\end{tabular}

The state transition model assumes the target moves at a constant velocity and is shown below as matrix $\mathrm{A}$, with the state vector $\mathrm{X}$ in Equation (3.4). Note $p_{x}, p_{y}$ and $v_{x}, v_{y}$ and the position and velocity of the target respectively.

$$
A=\left[\begin{array}{cccc}
1 & 0 & \Delta t & 0 \\
0 & 1 & 0 & \Delta t \\
0 & 0 & 1 & 0 \\
0 & 0 & 0 & 1
\end{array}\right], \hat{x}_{k}=\left[\begin{array}{c}
p_{x} \\
p_{y} \\
v_{x} \\
v_{y}
\end{array}\right]
$$

The observation model is based on the geometric position of the radar and the target state vector, $\hat{x}_{k}$, as shown in Figure 3.2 


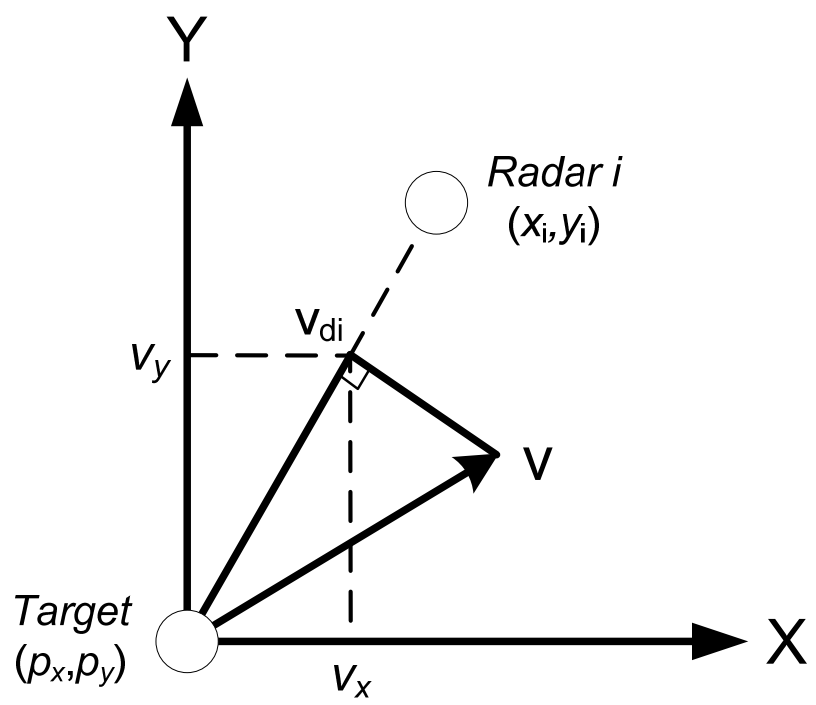

Figure 3.2: Shows the radar and target layout.

The observation model, which takes a state vector and gives the expected measurements, is described in Equation (3.5). Each $F_{i}$ represents an expected sensor node measurement, the Doppler velocity, shown as $v_{d i}$ in Figure 3.2

$$
F=\left[\begin{array}{c}
F_{1} \\
\vdots \\
F_{i}
\end{array}\right], F_{i}=\frac{v_{x}\left(x_{i}-p_{x}\right)+v_{y}\left(y_{i}-p_{y}\right)}{\sqrt{\left(x_{i}-p_{x}\right)^{2}+\left(y_{i}-p_{y}\right)^{2}}}
$$

To calculate the Kalman gain matrix $\mathrm{K}_{\mathrm{k}}$ the Jacobian matrix, $\mathrm{J}_{\mathrm{k}}$, of the observation model $\mathrm{F}$ is show in Equations (3.6)-(3.10)

$$
\begin{aligned}
& \frac{\partial F_{i}}{\partial p_{x}}=\frac{v_{x}\left(y_{i}-p_{y}\right)\left(x_{i}-p_{x}\right)-v_{y}\left(x_{i}-p_{x}\right)^{2}}{\left(\left(x_{i}-p_{x}\right)^{2}+\left(y_{i}-p_{y}\right)^{2}\right)^{\frac{3}{2}}} \\
& \frac{\partial F_{i}}{\partial p_{y}}=\frac{v_{y}\left(x_{i}-p_{x}\right)\left(y_{i}-p_{y}\right)-v_{x}\left(y_{i}-p_{y}\right)^{2}}{\left(\left(x_{i}-p_{x}\right)^{2}+\left(y_{i}-p_{y}\right)^{2}\right)^{\frac{3}{2}}} \\
& \frac{\partial F_{i}}{\partial v_{x}}=\frac{\left(x_{i}-p_{x}\right)}{\sqrt{\left(x_{i}-p_{x}\right)^{2}+\left(y_{i}-p_{y}\right)^{2}}} \\
& \frac{\partial F_{i}}{\partial v_{y}}=\frac{\left(y_{i}-p_{y}\right)}{\sqrt{\left(x_{i}-p_{x}\right)^{2}+\left(y_{i}-p_{y}\right)^{2}}}
\end{aligned}
$$




$$
J_{k}=\nabla F=\nabla\left[\begin{array}{c}
F_{1} \\
\vdots \\
F_{i}
\end{array}\right]=\left[\begin{array}{cccc}
\frac{\partial F_{1}}{\partial p_{x}} & \frac{\partial F_{1}}{\partial p_{y}} & \frac{\partial F_{1}}{\partial v_{x}} & \frac{\partial F_{1}}{\partial v_{y}} \\
\vdots & \vdots & \vdots & \vdots \\
\frac{\partial F_{i}}{\partial p_{x}} & \frac{\partial F_{i}}{\partial p_{y}} & \frac{\partial F_{i}}{\partial v_{x}} & \frac{\partial F_{i}}{\partial v_{y}}
\end{array}\right]
$$

The noise covariance matrices $\mathrm{Q}$ and $\mathrm{R}$ for the state transition model and observation model respectively are shown below in Equation (3.11)

$$
Q=\left[\begin{array}{cccc}
50 & 0 & \Delta t & 0 \\
0 & 50 & 0 & \Delta t \\
0 & 0 & 50 & 0 \\
0 & 0 & 0 & 50
\end{array}\right], R=\left[\begin{array}{cc}
20 & 0 \\
0 & 20
\end{array}\right]
$$




\section{Chapter 4 Implementation}

Show below in Figure 4.1 is the system block diagram of my implementation to the WSN tracking platform from Chapter 3.

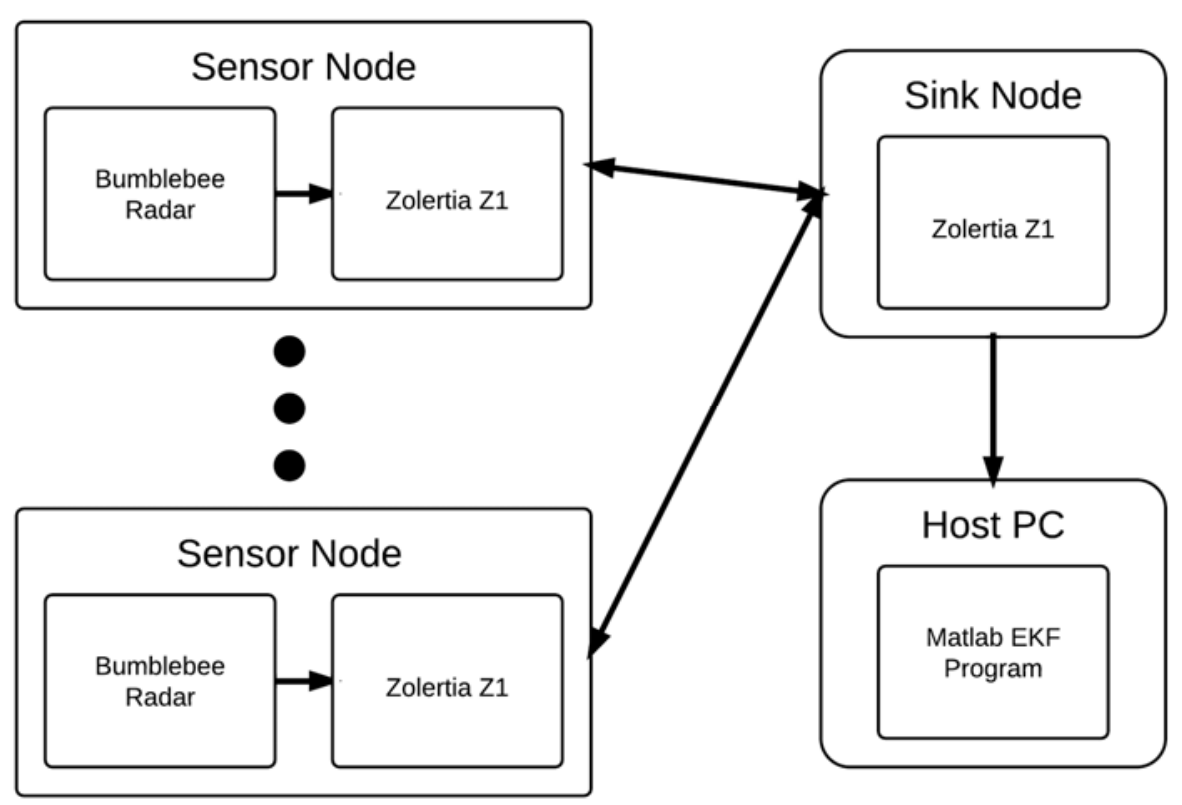

Figure 4.1: System Block Diagram of the tracking WSN.

\subsection{Sensing}

\subsubsection{Hardware}

The sensing hardware is implemented on two WSN node platforms the Tmote Sky mote and Zolertia Z1 shown in Figure 4.2 [13, 14]. Each sensor node and sink node can use either platform due to the portability of the Contiki software described in the next section. As mentioned in Chapter 3 the BumbleBee Radar 
by The Samraksh Company is connected to each sensor node [15]. Shown in Table 4.1 is the wiring for the Zolertia Z1 platform to the BumbleBee radar.

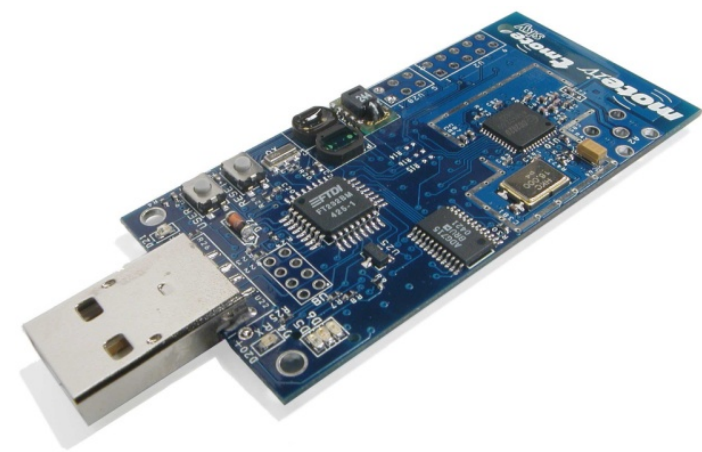

(a) Tmote Sky

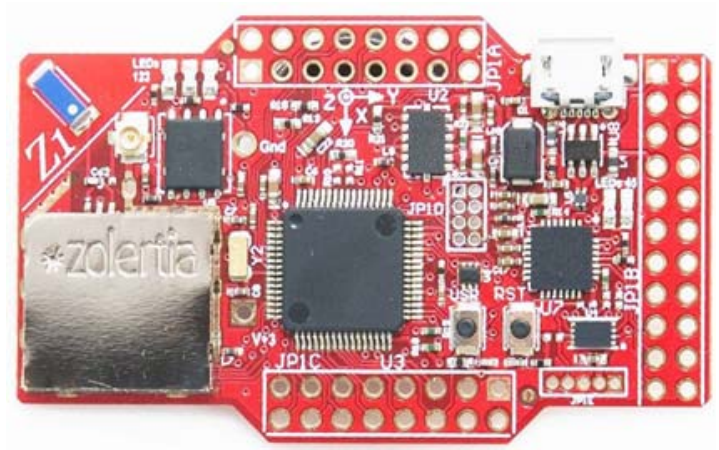

(b) Zolertia z1

Figure 4.2: The two WSN hardware Platforms used.

Table 4.1: Wiring for Sensor Node.

\begin{tabular}{|c|c|c|}
\hline BumbleBee Radar & Zolertia Z1 & Battery Pack \\
\hline J1-1 & & Ground \\
\hline J1-2 & & Power \\
\hline J1-3 & & Power \\
\hline J 1-4 & JP1A-2 & \\
\hline J2-1 & JP1A-1 & \\
\hline J2-2 & JP1A-7 & \\
\hline J2-4 & JP1A-9 & \\
\hline
\end{tabular}

\subsubsection{Software}

Each sensing node and the sink node runs Contiki an embedded OS. Contiki was chosen for its small footprint and large feature set. Contiki uses an event driven multi-tasking scheduler using a unique programming construct the Protothread. Protothreads are a memory efficient, stackless version of a thread that use cooperative scheduling allowing straight forward coding even with multiple 
threads. Contiki also provides a lightweight IP stack library to ease implementation of networking.

The client.c program shown in Appendix A runs on each sensor node and behaves as shown below in Figure 4.3. The first event thread simple initializes the sensor node by configuring the radar driver, creating the UDP network connections, and starting the periodic timer. The second event thread is based around the periodic timer which creates a paced loop that reads the radar sensor and computes the autocorrelation. The last two event threads are used to receive and then respond to beacon messages from the sink node.
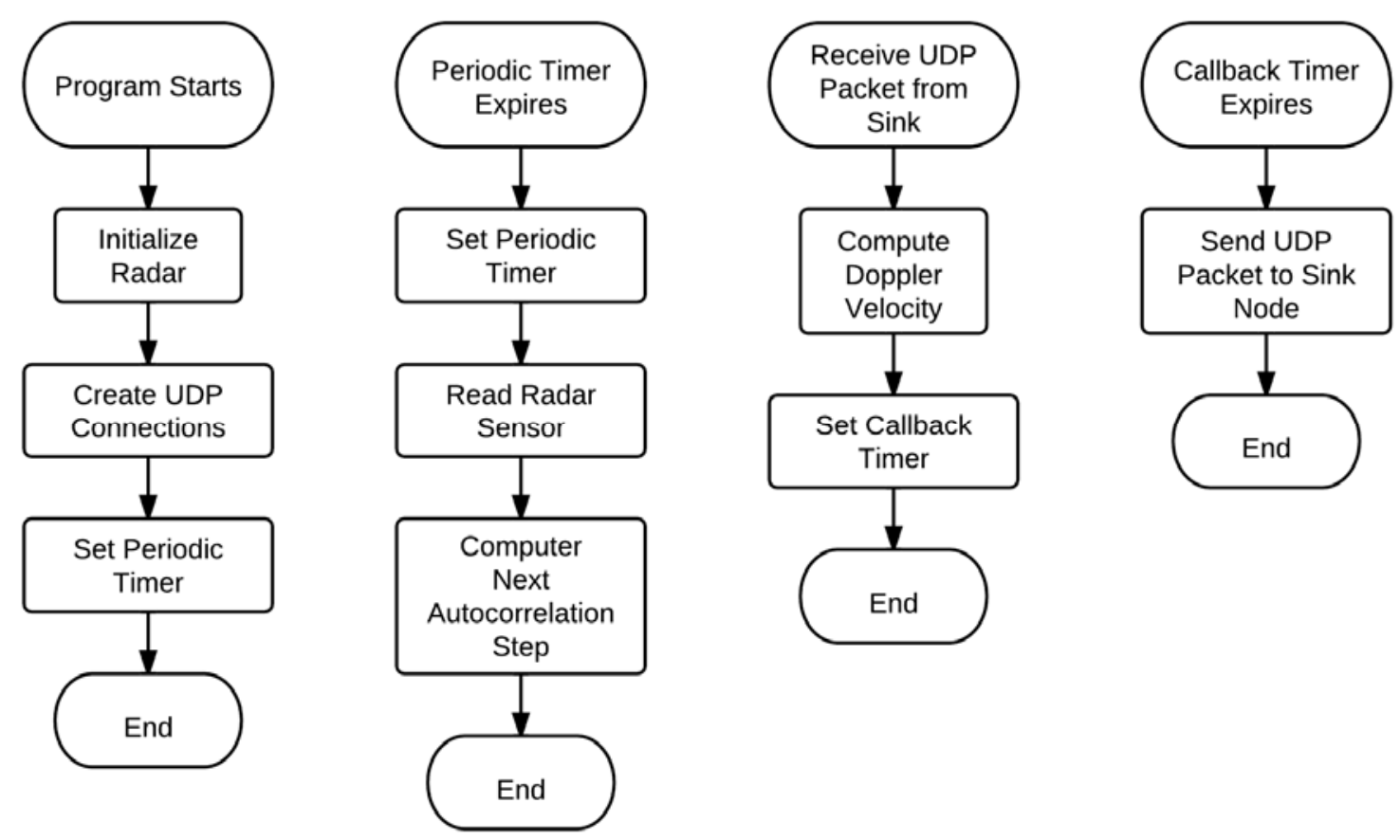

Figure 4.3: Block Diagram of client.c code flow.

The sink node runs the host.c program shown in Appendix A and is described below in Figure 4.4. In the sink node the first event thread initializes by creating the UDP network connections and starting the periodic callback timer for the beacon. The second event thread sends the periodic beacon messages over the UDP connection to all sensor nodes. The last event thread receives data from the sensor nodes and prints it to the serial link sent to the host PC. 


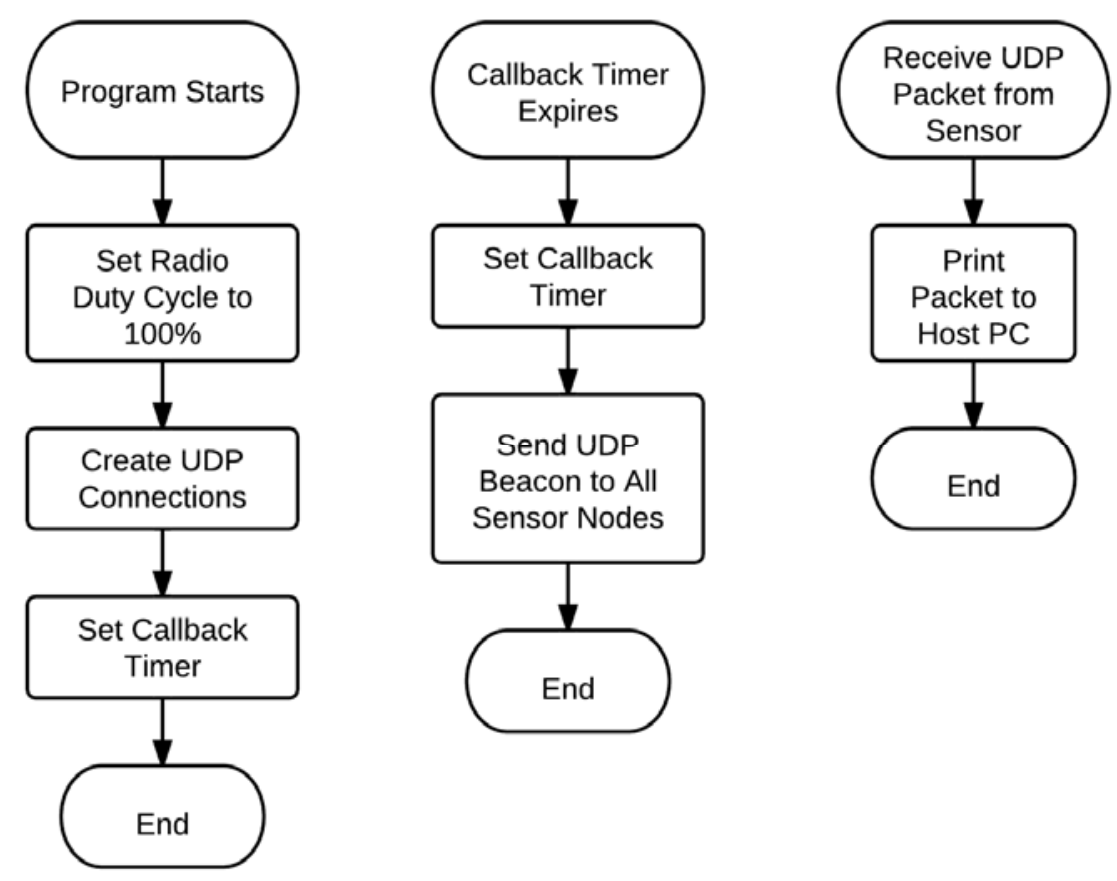

Figure 4.4: Block Diagram of host.c code flow.

\subsection{Tracking}

The host PC that is running Matlab $(\mathbb{C}$ and performs the tracking is a Pentium4 2.6GHz with $1 \mathrm{G}$ ram. The Matlab $\odot$ code tracker.m is shown in Appendix A and behaves as shown in Figure 4.5. The program starts by initializing the EKF parameters and setting up the serial link to the sink node. The serial link is then read and sensor data is matched into set corresponding to each beacon. Since the beacon reaches each sensor at approximately the same time this beacon set has the time synchronous readings of each sensor radar. Once a set is completed it is run through the EKF and the results are displayed. Since the UDP network is best effort not all messages are guaranteed to be received. The time step for the EKF is dynamic and is iterated by the time difference between two complete beacon sets. This allows the tracking to be robust even in a lossy WSN. 


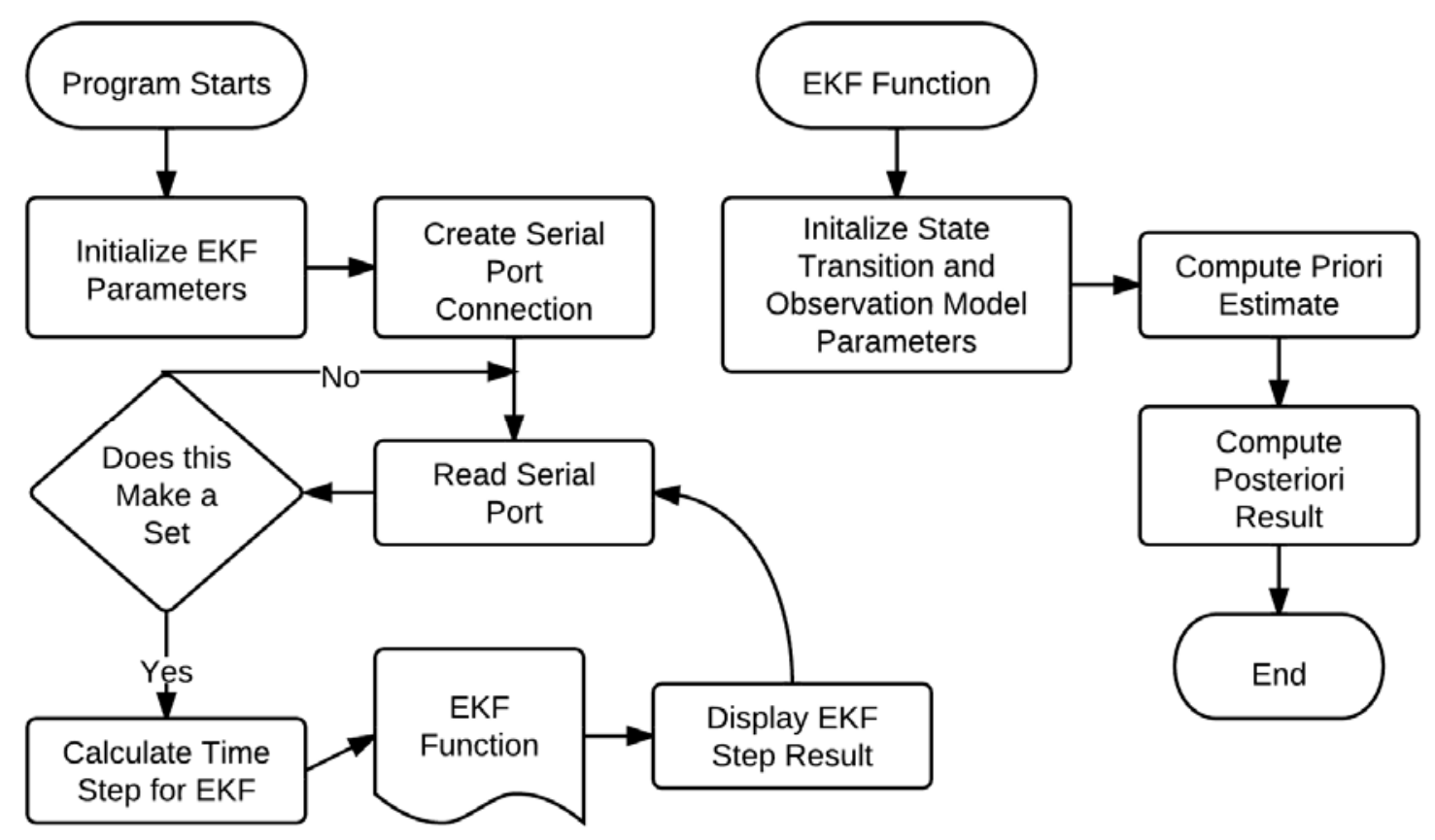

Figure 4.5: Block diagram of tracker.m program flow. 


\section{Chapter 5 Evaluation}

To evaluate the accuracy of the implementation a simulation was and simple test bed were setup to evaluate performance of the system. Shown in Figure 5.1 is the configuration used for both the simulation and tests, which shows the two sensors 6 ' apart with the sink node in the middle all collinear.

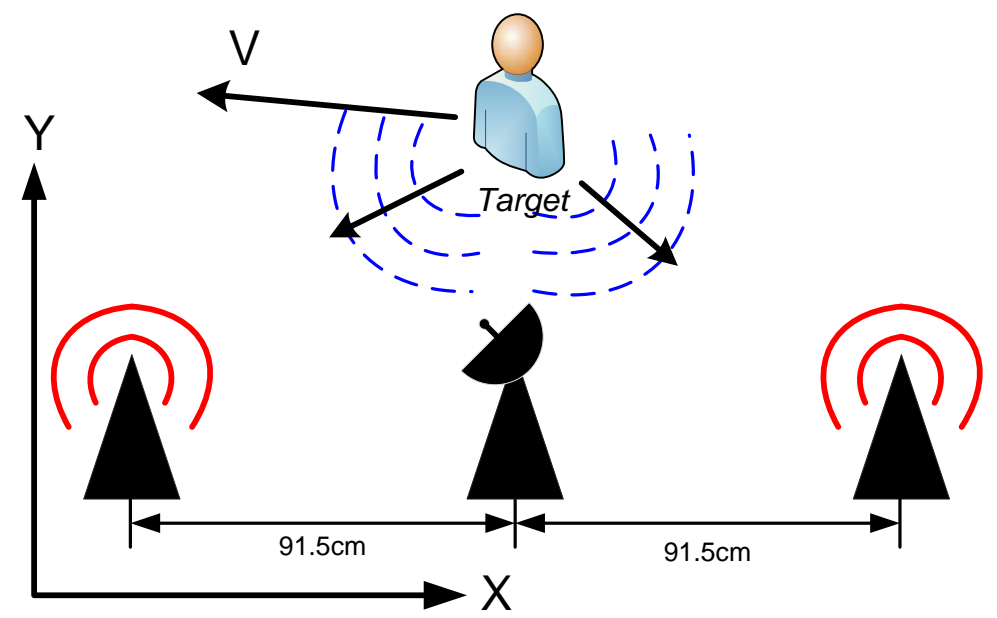

Figure 5.1: Diagram of experiment and simulation layout.

\subsection{Experiment}

Both radars' ranges were adjusted to full-scale $(10 \mathrm{~m})$ and then a single subject moved in the WSN in a predetermined pattern so the ground truth was known. The sink node was set to send beacon messages every 0.5 seconds. The tests were performed in a furnished graduate student office with only the subject moving at approximately $30 \mathrm{~cm} / \mathrm{s}$. Three different test patterns were used; a line perpendicular to the two sensors, an oblique line, and a diamond. Shown in

Figure 5.2 are the test results for the perpendicular pattern. 

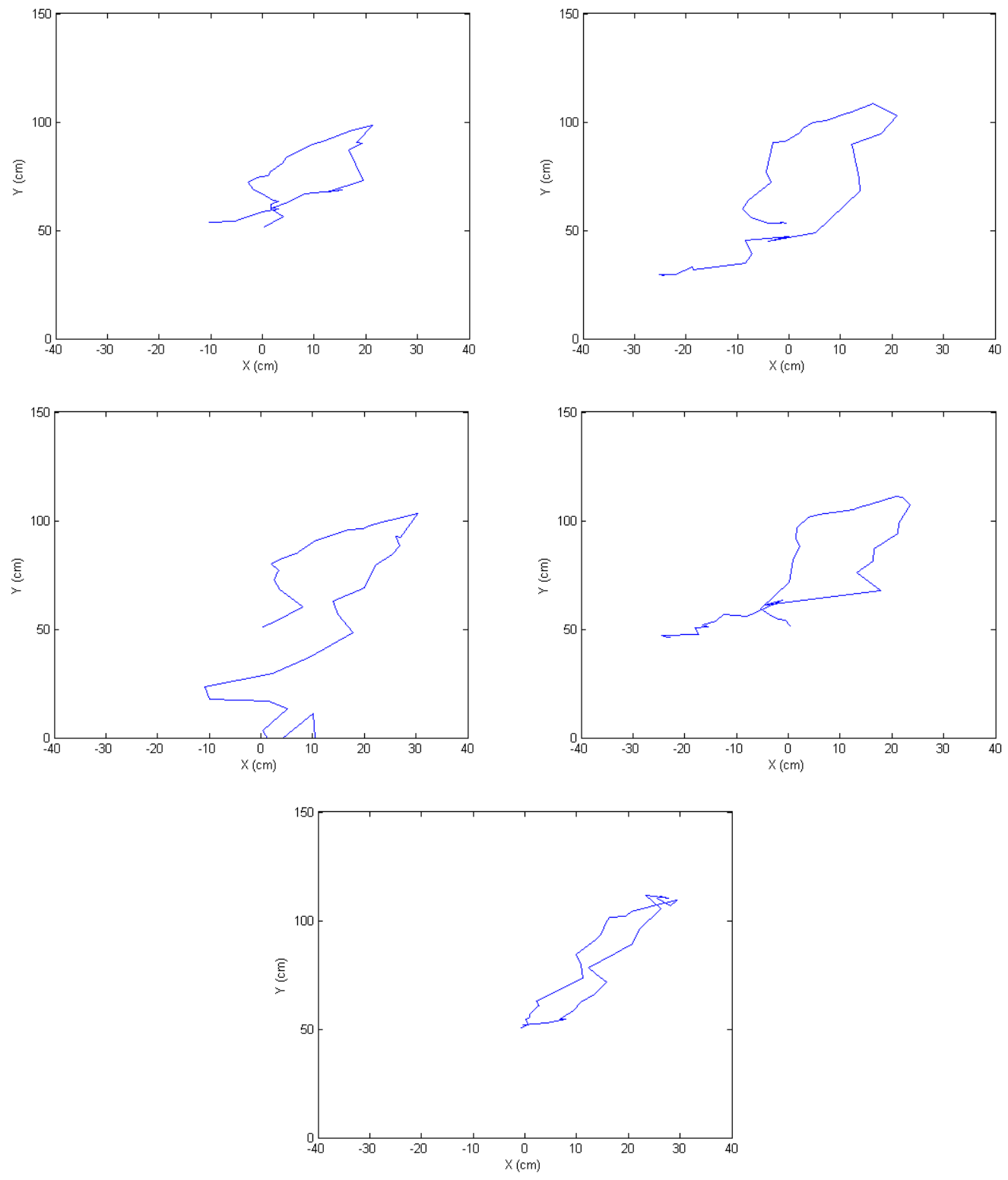

Figure 5.2: Experimental results for the perpendicular test pattern.

The perpendicular test pattern was created by starting $50 \mathrm{~cm}$ back from the $\mathrm{x}$ axis and on the $\mathrm{y}$ axis. The subject then moved $100 \mathrm{~cm}$ forward and then $100 \mathrm{~cm}$ back to the starting position. It can be seen from

Figure 5.2 the EKF generally tracked the trajectory of the subject was close to linear in shape. In all five trials the EKF does not track the subject to the expected $150 \mathrm{~cm}$ but does generally finish near the place it started. Shown in Figure 5.3 are the results from the oblique line test trials. 

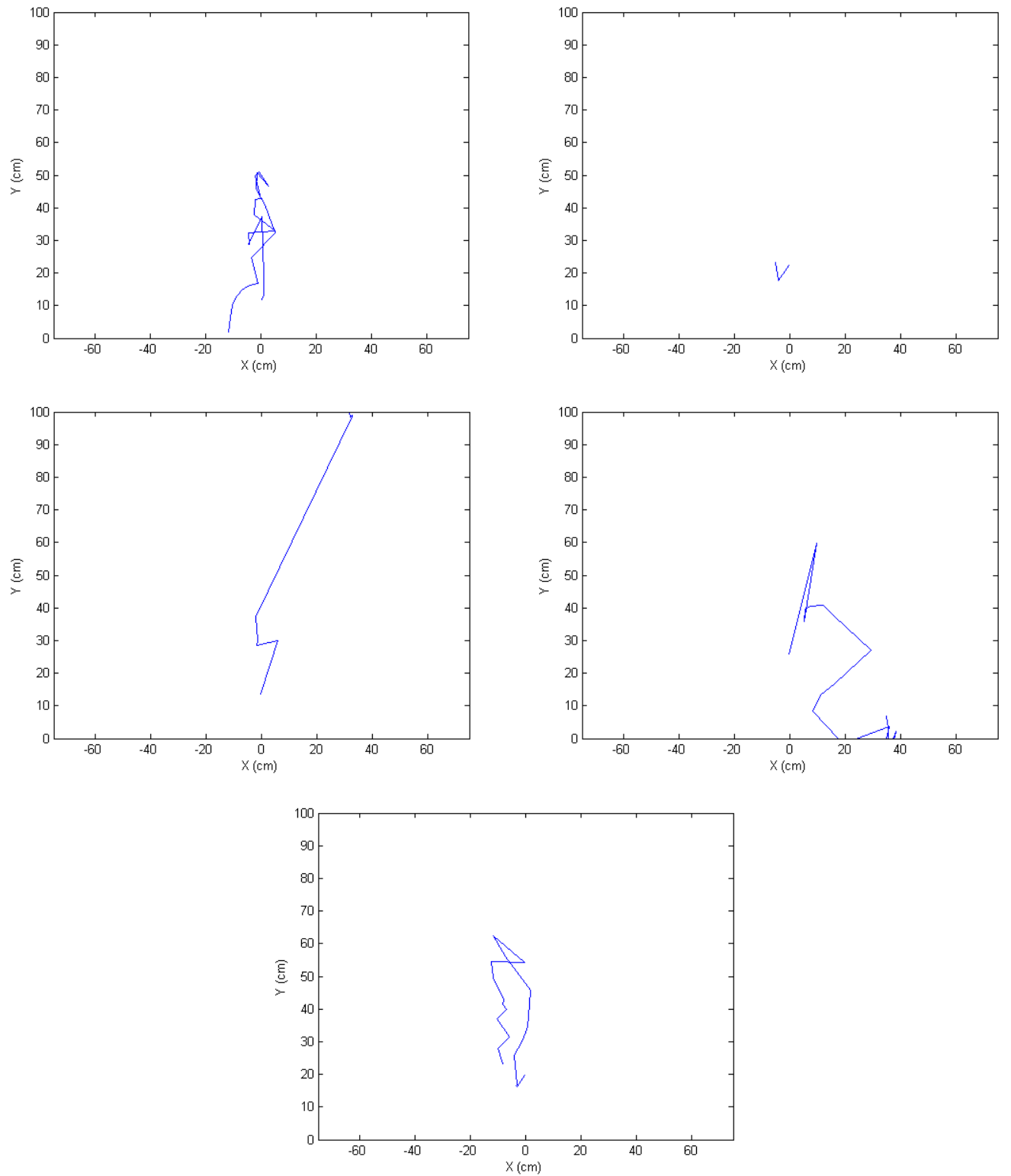

Figure 5.3: Experimental results for the oblique test pattern.

The oblique test pattern is made by the subject starting $10 \mathrm{~cm}$ from the $\mathrm{x}$ axis and on the y axis. The subject then moves $75 \mathrm{~cm}$ along the y axis and -75 along the $\mathrm{x}$ axis to create an oblique line to the $\mathrm{x}$ and $\mathrm{y}$ axes. All trials have a general linear shape but again do not track all the way to the far point of the test. I attribute this to the noise threshold filter in the EKF which is set to $5 \mathrm{~cm} / \mathrm{s}$. Shown in Figure 5.4 are some of the trials from the diamond test pattern. 

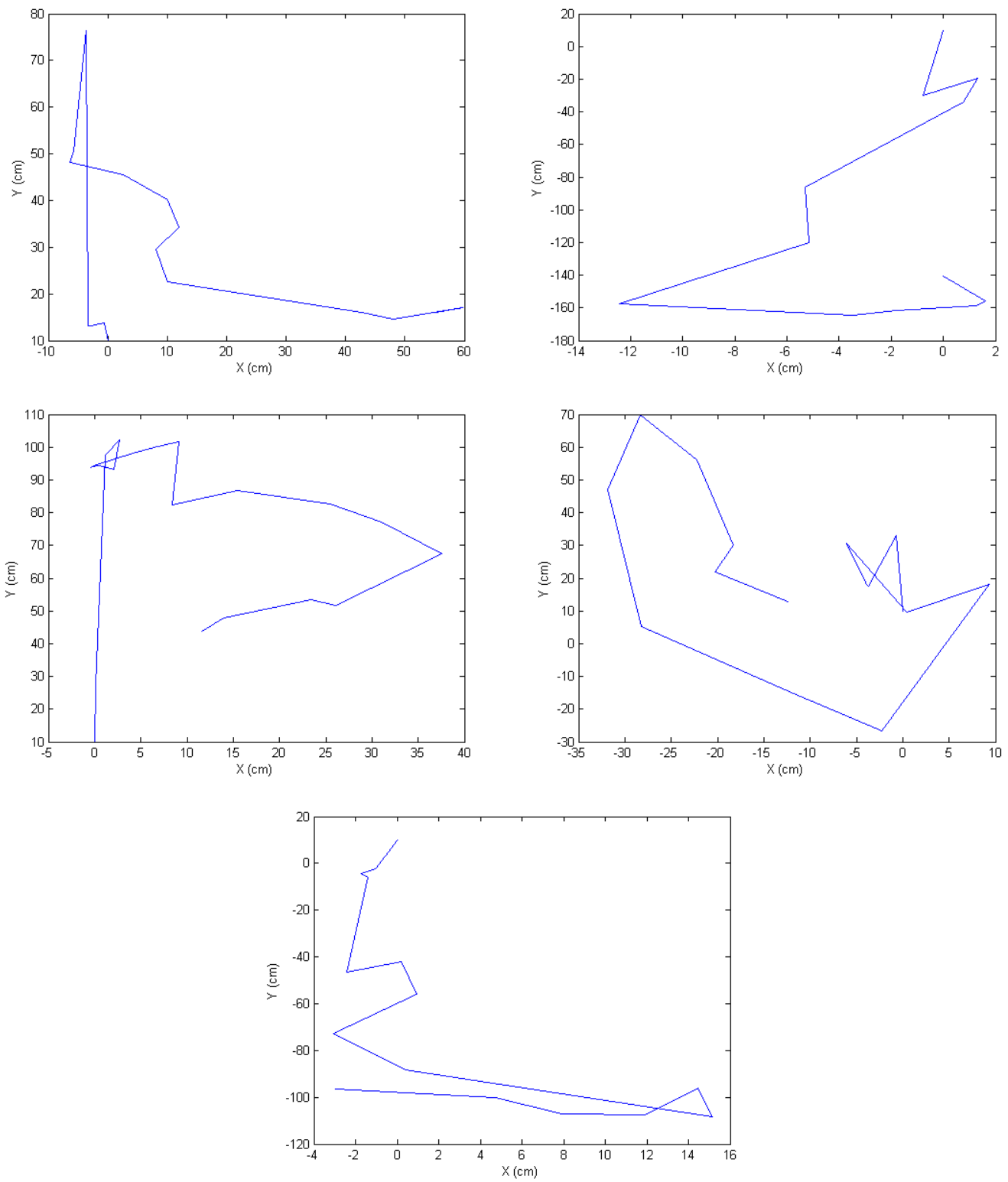

Figure 5.4: Experimental results for the diamond test pattern.

To generate the diamond test pattern the subject started $10 \mathrm{~cm}$ from the $\mathrm{x}$ axis and on the y axis, same as the oblique pattern tests. The subject then moved in $140 \mathrm{~cm}$ by $140 \mathrm{~cm}$ segments to create a square diamond pattern. The failure of the EKF is apparent from the lack of a diamond shaped pattern and will be discussed in Chapter 6. 


\subsection{Simulation}

Shown in Figure 5.5 are the simulation results for the perpendicular test pattern.
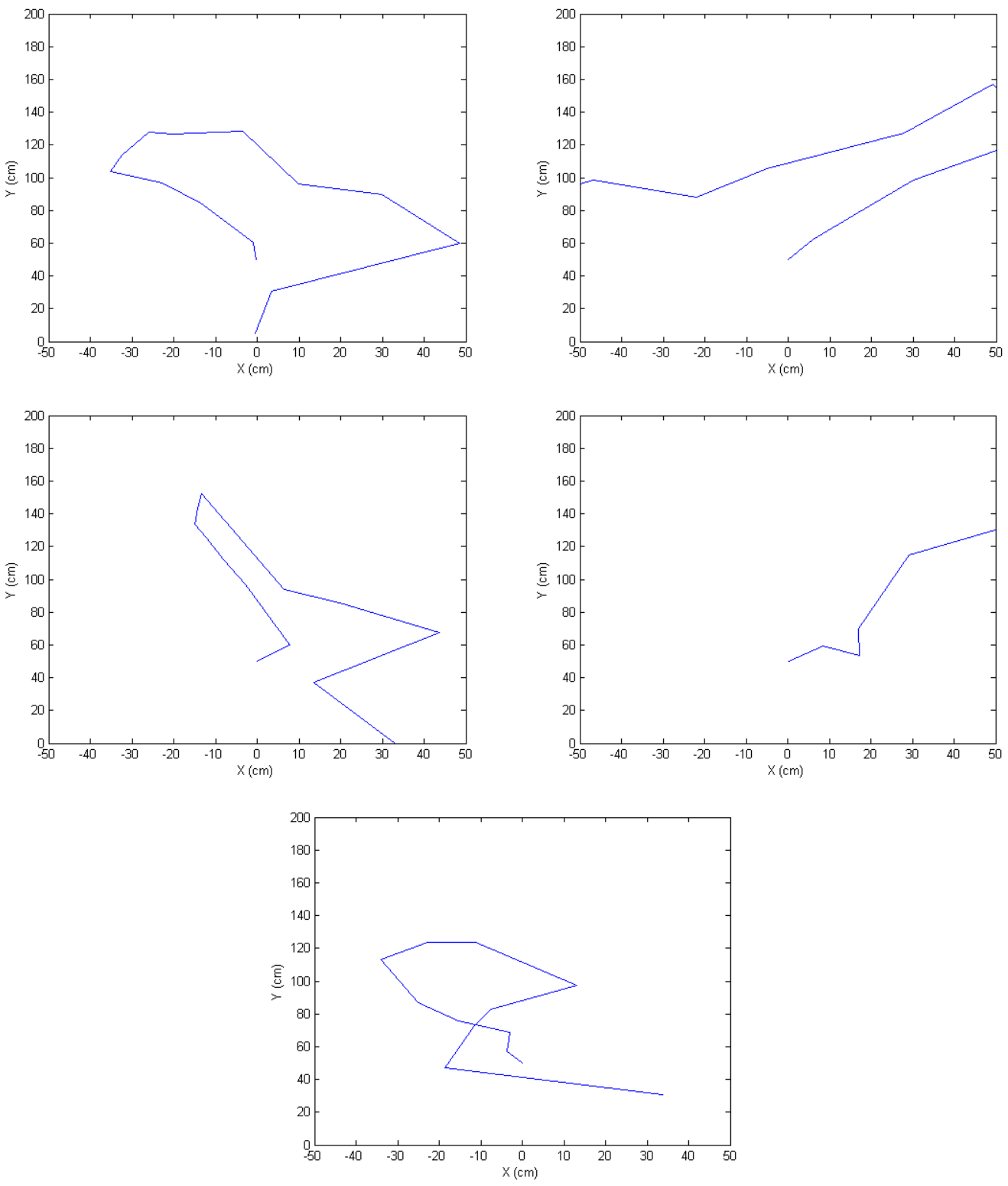

Figure 5.5: Simulation results of perpendicular test pattern. 
These results are visually comparable to the experiment results for the perpendicular test pattern. The results for the oblique test pattern also show about the same performance as seen from the experimental test results.
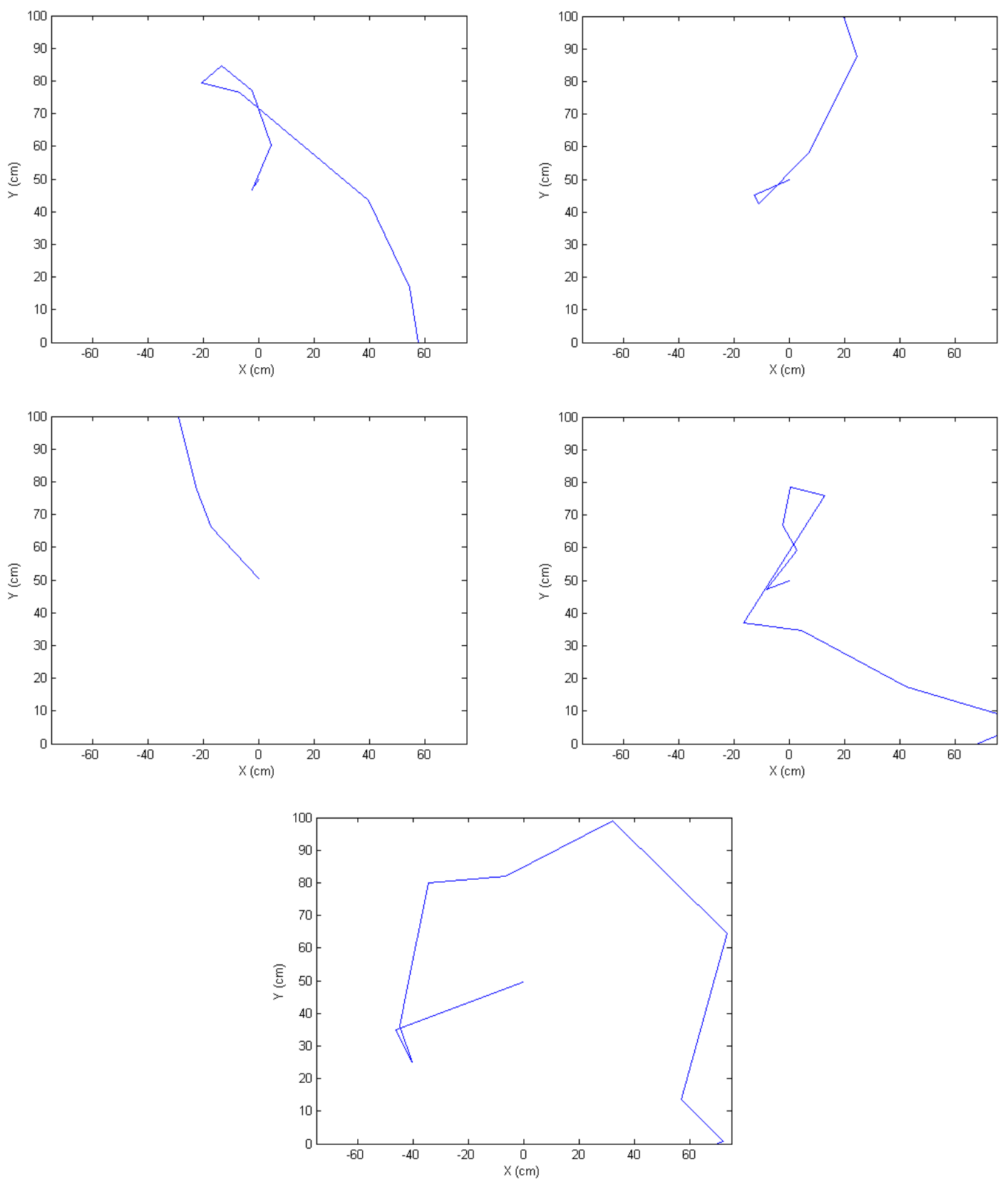

Figure 5.6: Simulation results of oblique test pattern. 


\section{Chapter 6 Discussion}

Although the EKF runs in real-time each step was logged for data analysis. Shown in Table 6.1 are the statistical results from the experiment for the perpendicular test pattern.

Table 6.1: Statistical summary for experimental perpendicular test pattern.

\begin{tabular}{|l|c|c|c|c|c|c|c|}
\hline Trial & 1 & 2 & 3 & 4 & 5 & Average & Std Dev \\
\hline Average Error $(\mathrm{cm})$ & 30.3 & 33.6 & 39.8 & 28.5 & 24.9 & 31.4 & 5.6 \\
\hline Max Error $(\mathrm{cm})$ & 56.9 & 59.9 & 66.4 & 57.3 & 49.6 & 58.0 & 6.1 \\
\hline
\end{tabular}

Following the results from Table 6.1 we are 95\% confident the average error of the tracking algorithm is within $31.4 \pm 11.2 \mathrm{~cm}$, which gives a resolution of approximately 0.5 meters. Furthermore, we are 95\% confident the maximum error is $58.0 \pm 12.2 \mathrm{~cm}$. The maximum error is also approximately 0.5 meters.

Table 6.2: Statistical summary for experimental oblique test pattern.

\begin{tabular}{|l|c|c|c|c|c|c|c|}
\hline Trial & 1 & 2 & 3 & 4 & 5 & Average & Std Dev \\
\hline Average Error $(\mathrm{cm})$ & 42.8 & 43.7 & 94.2 & 72.2 & 39.0 & 58.4 & 24.0 \\
\hline Max Error $(\mathrm{cm})$ & 89.9 & 97.7 & 126.0 & 116.1 & 86.3 & 103.2 & 17.2 \\
\hline
\end{tabular}

The results from the oblique test pattern are close to the perpendicular but with higher averages and larger standard deviations. We are 95\% confident the average error is $58.4 \pm 48.0 \mathrm{~cm}$. This resolution is approximately 1 meter. We are 
$95 \%$ confident the maximum error is $103.2 \pm 34.4 \mathrm{~cm}$, which is close to the 1 meter but we are much less certain.

The diamond test was not worth statistically analyzing because it can be seen from Figure 5.4 the algorithm did not track a diamond shape. This is because of the constant velocity assumption and low sample rate of the sensor node by the beacon. The largest limitation of this WSN setup is the time step of the EKF. The current network setup cannot reduce the time step of the beacon to less than half a second. Any faster and the network saturates with messages causing messages to be lost and/or received out of order. This would cause unreasonable delays to the real-time aspect of the EKF. 


\section{Chapter 7 Future Work}

Even though the time step of the current implementation places limits on the performance of tracking there are other ways to gain performance such as changing the tracking algorithm.

The plain EKF was implemented out of simplicity and several other versions exist such as the unscented EKF. Even better than an EKF would be to implement a Particle Filter which would better represent the non-linear observation model leading to higher resolutions.

The current setup can only track a single target within its domain and this leads to a simpler implementation but limits the applications of the platform. Instead of using autocorrelation to determine the Doppler velocity of a target a Fast-Fourier Transform (FFT) could be used. Although this is much more computationally intensive it would allow for the tracking of many targets at one time. Our current EFK cannot work in conjunction with the FFT method because it only supports a single position hypothesis but a multi-hypothesis tracking algorithm such as a Particle Filter could be used. Multi-target tracking is a desirable feature and possible with the current hardware. 


\section{Chapter 8 Conclusion}

The goal of this report was to implement a tracking WSN platform similar to what was developed by Lim, Wang, and Terzis (1). A WSN was developed using the Tmote Sky, Zolertia Z1, and a host PC. Each sensor node consisted of a BumbleBee radar connected to a Zolertia Z1, which sent its data to a sink node. The sink node was a Tmote Sky connected to a host PC through a serial link. The host PC ran a Matlab@ program that read in the sensor data from the serial link and fused it through an EKF.

An experiment was setup to verify the WSN platform. Several test target movement patterns were used and the results logged for further analysis. It was found that simple linear motion could be tracked but any complex motion with numerous changes in velocity or direction failed to be tracked. This can be attributed to the state transition model assumption of constant velocity and the large time step size of the EKF. Results could be improved by changing the tracking algorithm to a different EKF such as an unscented EKF or using a Particle Filter to better model the observation model. Furthermore, our research was limited to single target tracking but could be expanded to multi-target tracking if autocorrelation was replaced with a FFT and using a multiple hypothesis tracking algorithm such as a Particle Filter. 


\section{Works Cited}

[1] L. J ong Hyun, I. J. Wang, and A. Terzis, "Tracking a non-cooperative mobile target using low-power pulsed Doppler radars," in Local Computer Networks (LCN), 2010 IEEE 35th Conference on, 2010, pp. 913-920.

[2] W. Yun, W. Xiaodong, X. Bin, W. Demin, and D. P. Agrawal, "Intrusion Detection in Homogeneous and Heterogeneous Wireless Sensor Networks," Mobile Computing, IEEE Transactions on, vol. 7, pp. 698-711, 2008.

[3] T. Ali Maleki, K. Arezou, and A. Hamid, "Smart home care network using sensor fusion and distributed vision-based reasoning," in Proceedings of the 4th ACM international workshop on Video surveillance and sensor networks Santa Barbara, California, USA: ACM, 2006.

[4] P. Bahl and V. N. Padmanabhan, "RADAR: an in-building RF-based user location and tracking system," in INFOCOM 2000. Nineteenth Annual J oint Conference of the IEEE Computer and Communications Societies. Proceedings. IEEE, 2000, pp. 775-784 vol.2.

[5] S. Shenoy and T. J indong, "Simultaneous localization and mobile robot navigation in a hybrid sensor network," in Intelligent Robots and Systems, 2005. (IROS 2005). 2005 IEEE/ RSJ International Conference on, 2005, pp. 1636-1641.

[6] B. P. Nissanka, C. Anit, and B. Hari, "The Cricket location-support system," in Proceedings of the 6th annual international conference on Mobile computing and networking Boston, Massachusetts, United States: ACM, 2000.

[7] K. Branislav, L. Akos, and K. Xenofon, "Tracking mobile nodes using RF Doppler shifts," in Proceedings of the 5th international conference on Embedded networked sensor systems Sydney, Australia: ACM, 2007. 
[8] Y. Nishida, T. Hori, S. Murakami, and H. Mizoguchi, "Minimally privacyviolative system for locating human by ultrasonic radar embedded on ceiling," in Systems, Man and Cybernetics, 2004 IEEE International Conference on, 2004, pp. 1549-1554 vol.2.

[9] L. Hongbin, M. Di, C. J iming, S. Youxian, and S. Xuemin, "Networked Ultrasonic Sensors for Target Tracking: An Experimental Study," in Global Telecommunications Conference, 2009. GLOBECOM 2009. IEEE, 2009, pp. 1-6.

[10] J . M. Sanchez-Matamoros, J. R. M. d. Dios, and A. Ollero, "Cooperative localization and tracking with a camera-based WSN," in Mechatronics, 2009. ICM 2009. IEEE International Conference on, 2009, pp. 1-6.

[11] H. Tian, P. Vicaire, Y. Ting, L. Liqian, G. Lin, Z. Gang, R. Stoleru, C. Qing, J. A. Stankovic, and T. Abdelzaher, "Achieving Real-Time Target Tracking UsingWireless Sensor Networks," in Real-Time and Embedded Technology and Applications Symposium, 2006. Proceedings of the 12th IEEE, 2006, pp. 37-48.

[12] M. Xufei, T. ShaoJ ie, X. Xiaohua, L. Xiang-Yang, and M. Huadong, "iLight: Indoor device-free passive tracking using wireless sensor networks," in INFOCOM, 2011 Proceedings IEEE, 2011, pp. 281-285.

[13] "Tmote Sky Ultra low power IEEE 802.51.4 compliant wireless sensor module," Moteiv Corperation, 2006. Available from http:// www.sentilla.com/ files/ pdf/ eol/tmote-sky-datasheet.pdf

[14] "Z1 Datasheet," Zolertia, 2010. Available from http:/ / zolertia.com/ sites/ default/ files/ Zolertia-Z1-Datasheet.pdf

[15] "Users manual for the BumbleBee (Model 0)," The Samraksh Company, 2008. Available from http:// www.samraksh.com/ docs/ BumbleBee-UMv103.pdf 


\section{Appendix A Software}

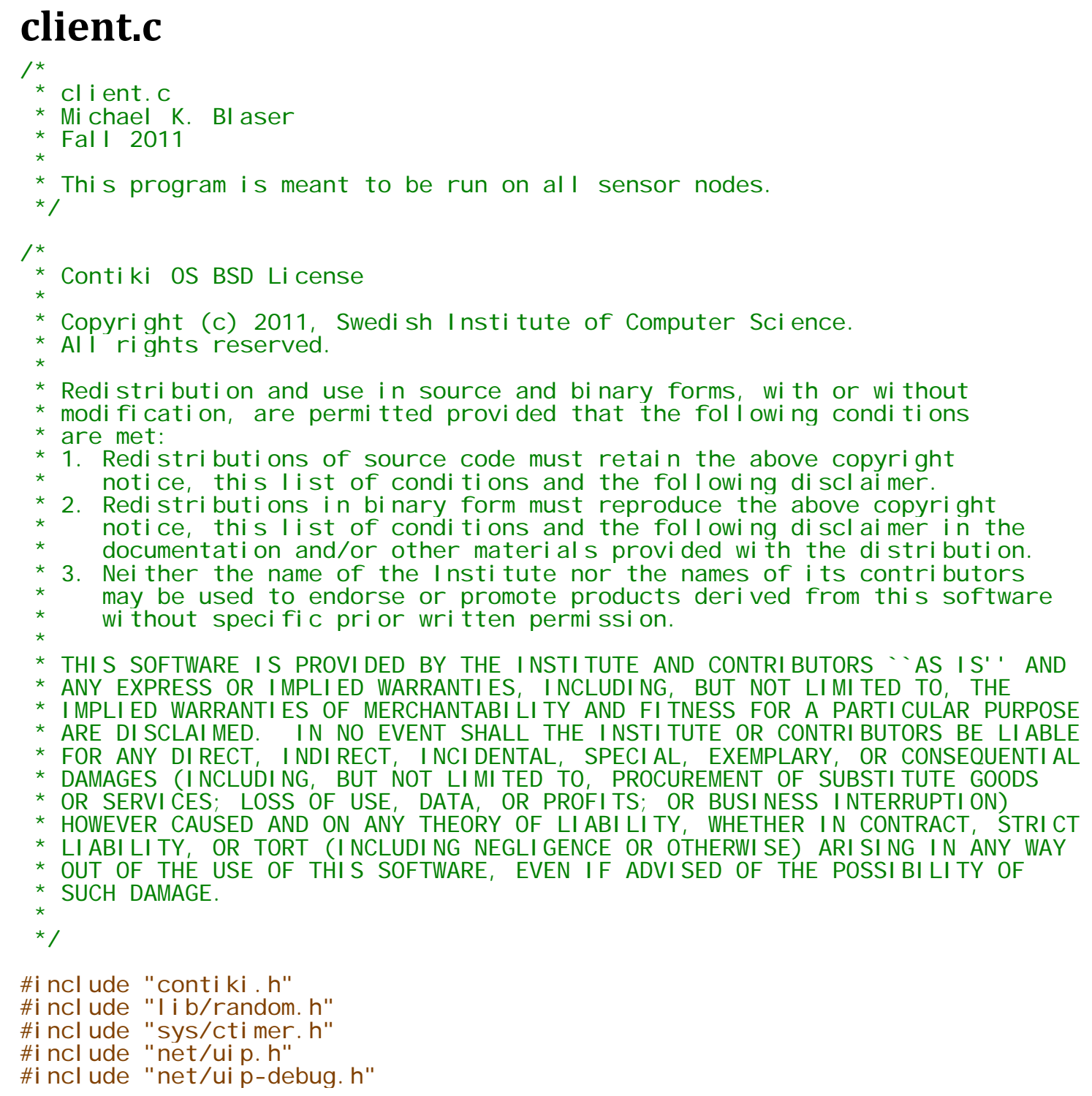

A-1 


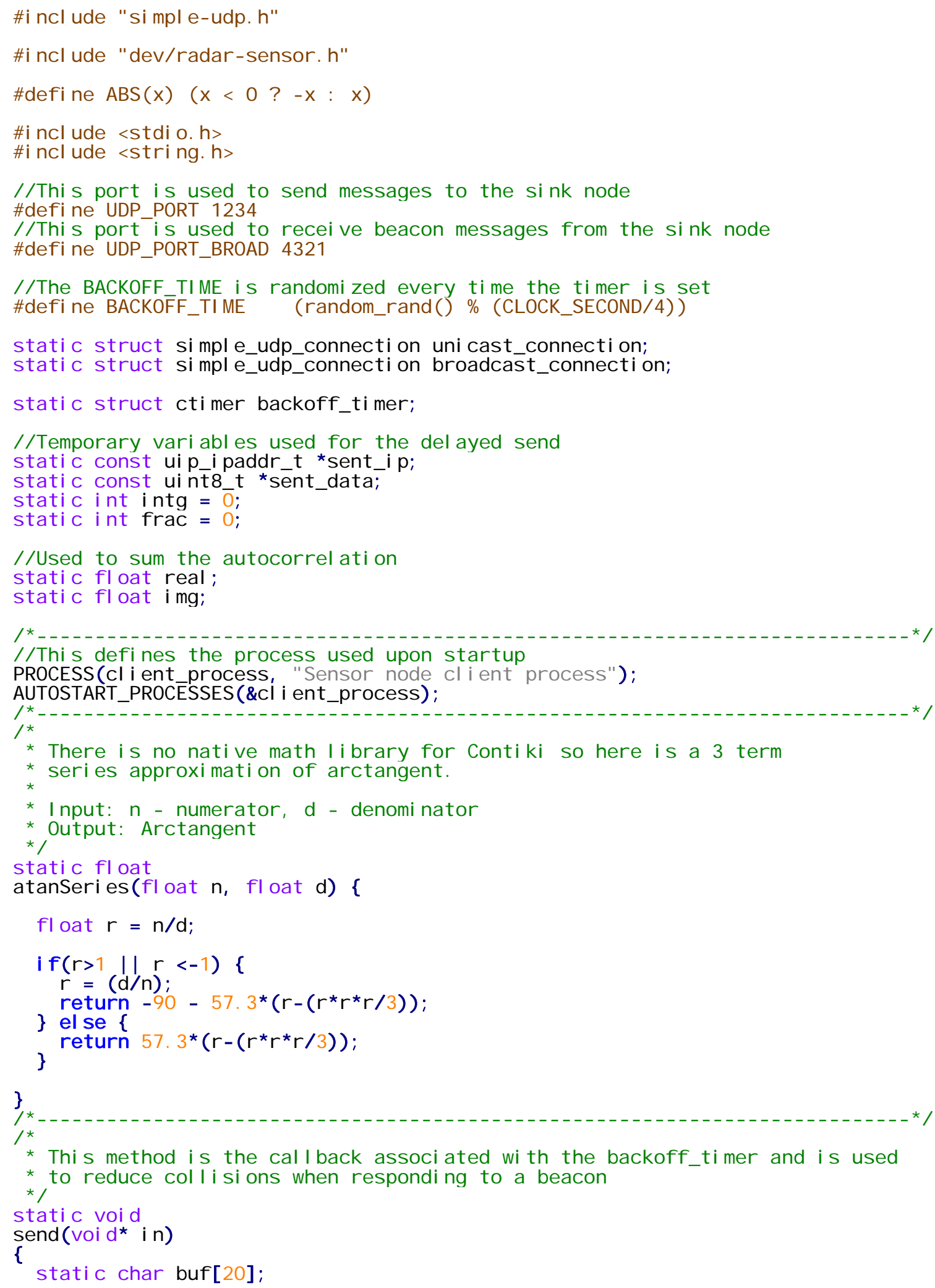




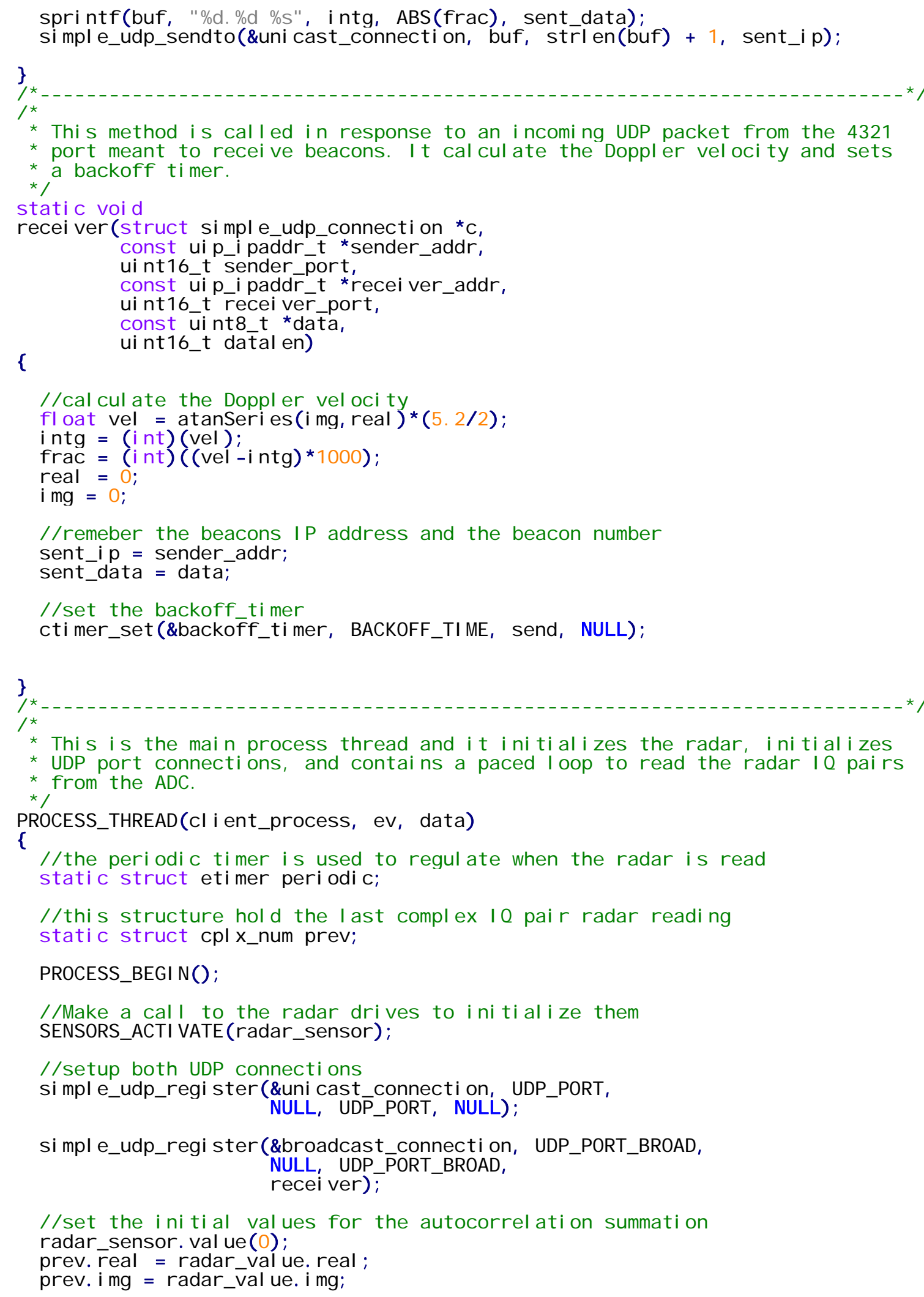




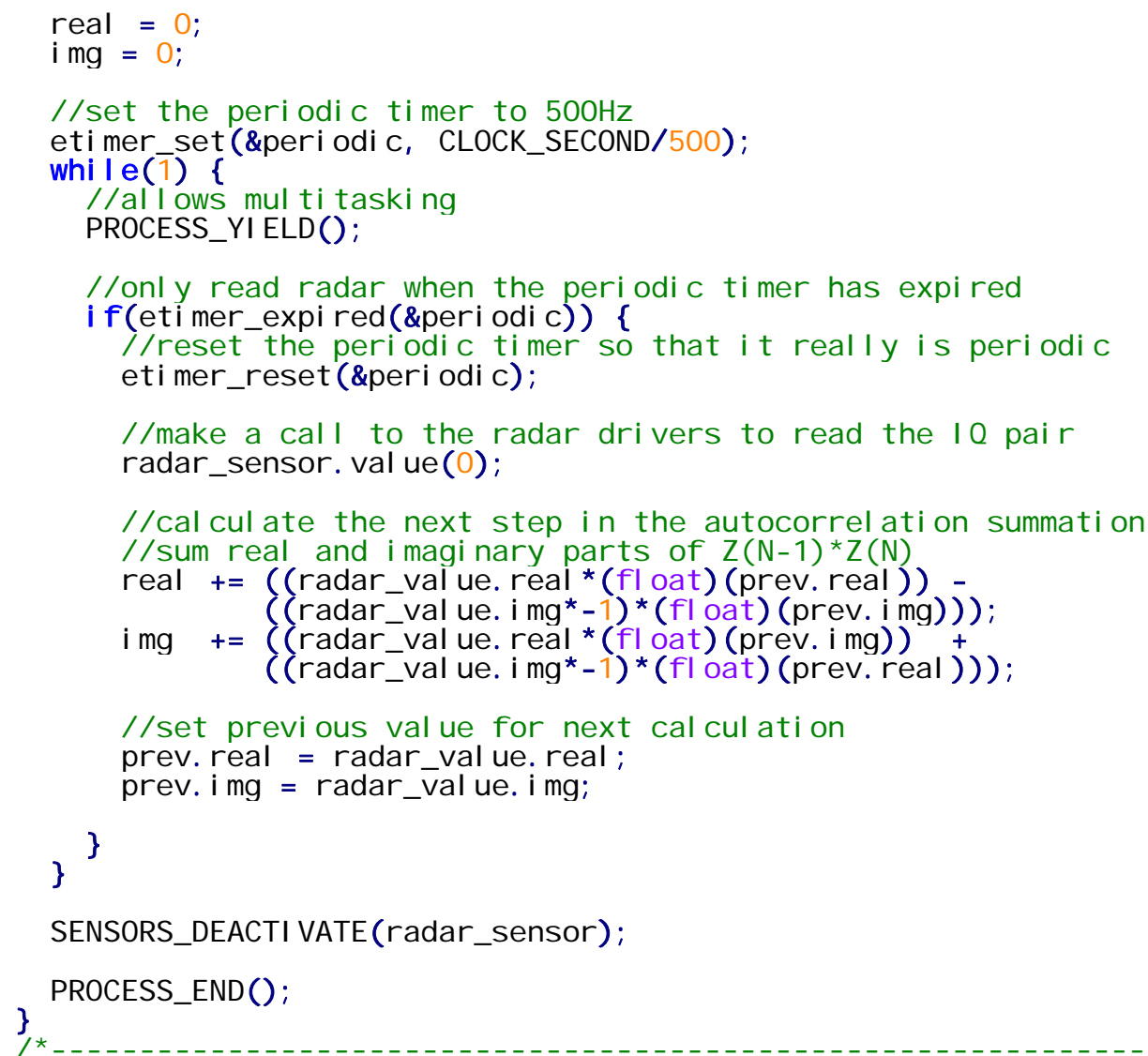




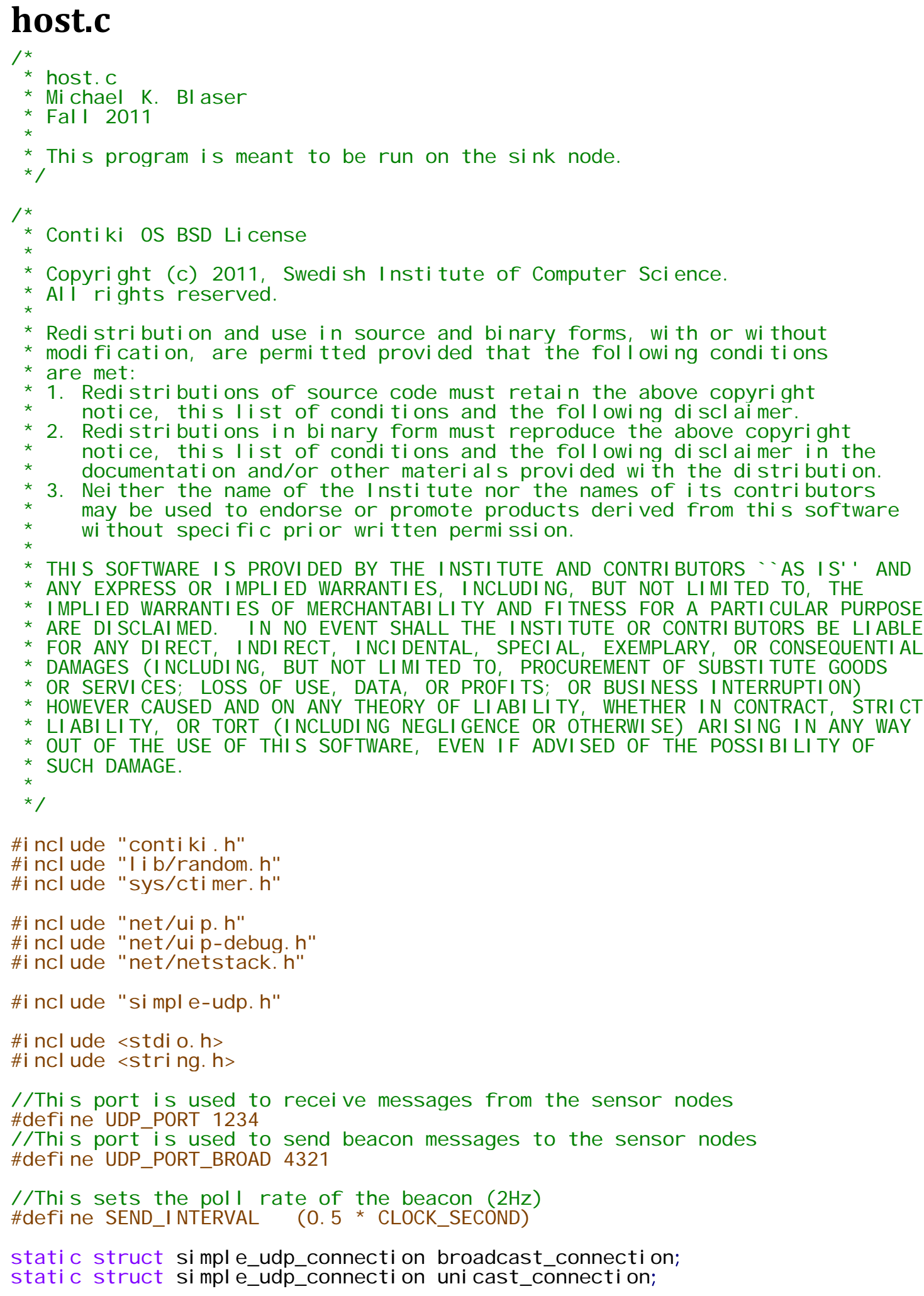

A-5 


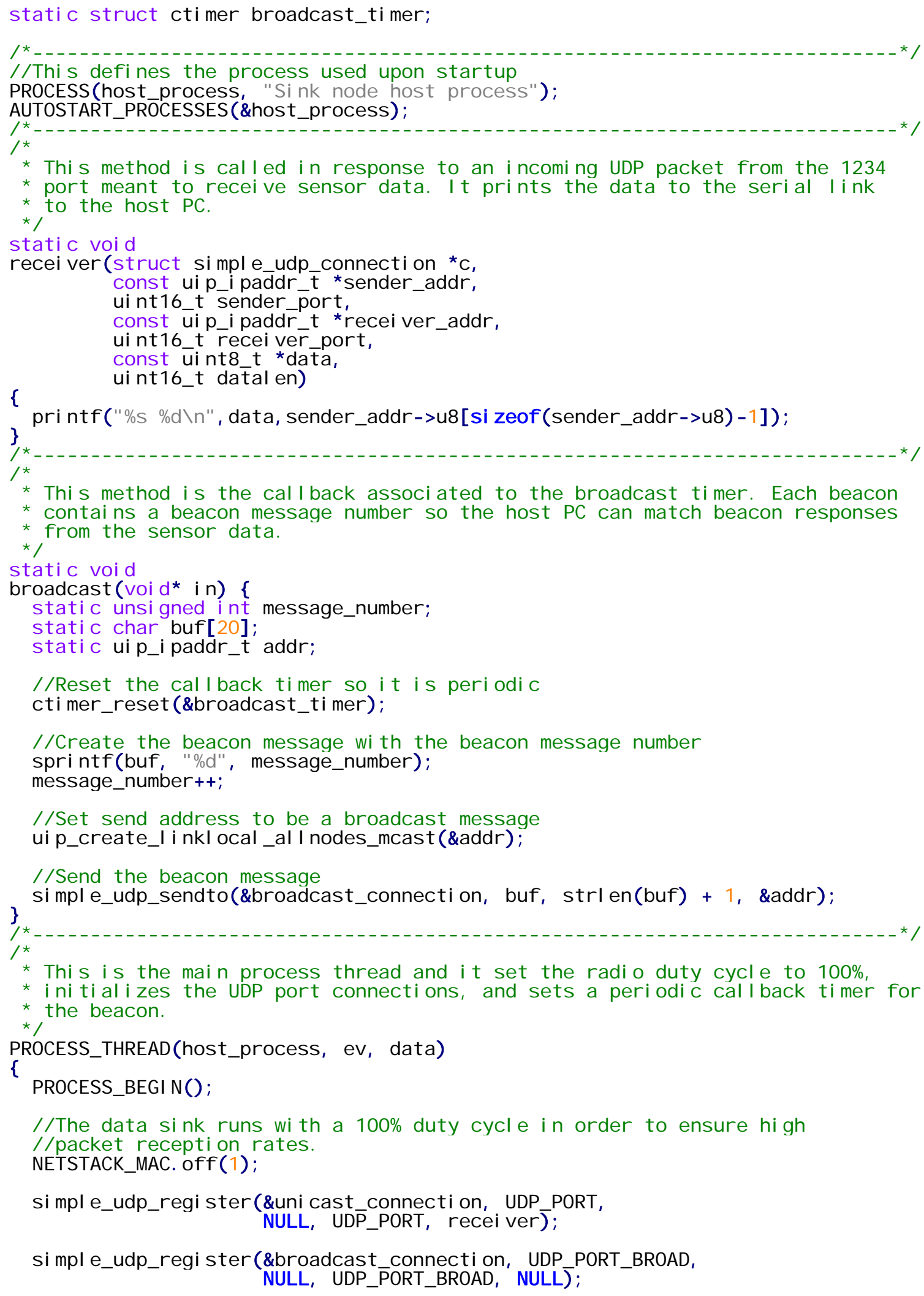




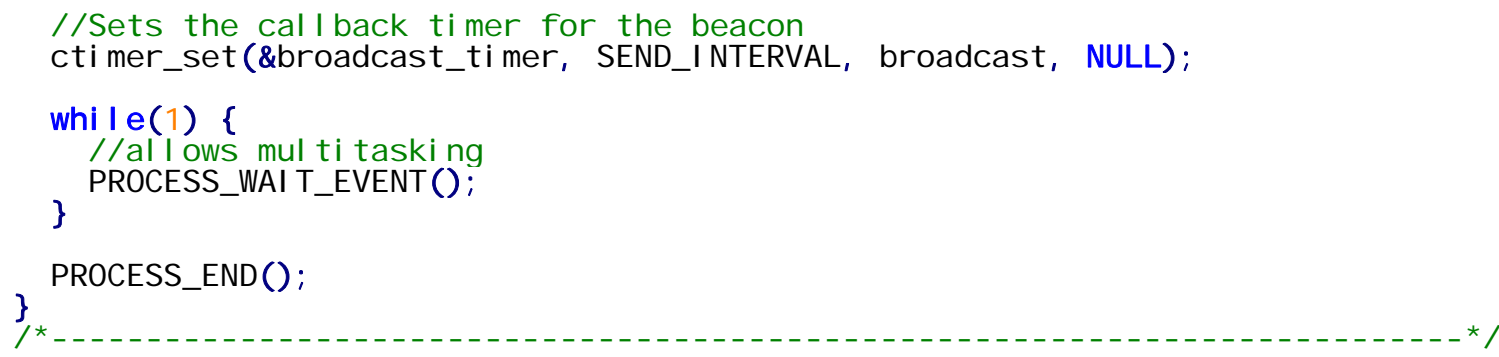




\section{radar-sensor.h}

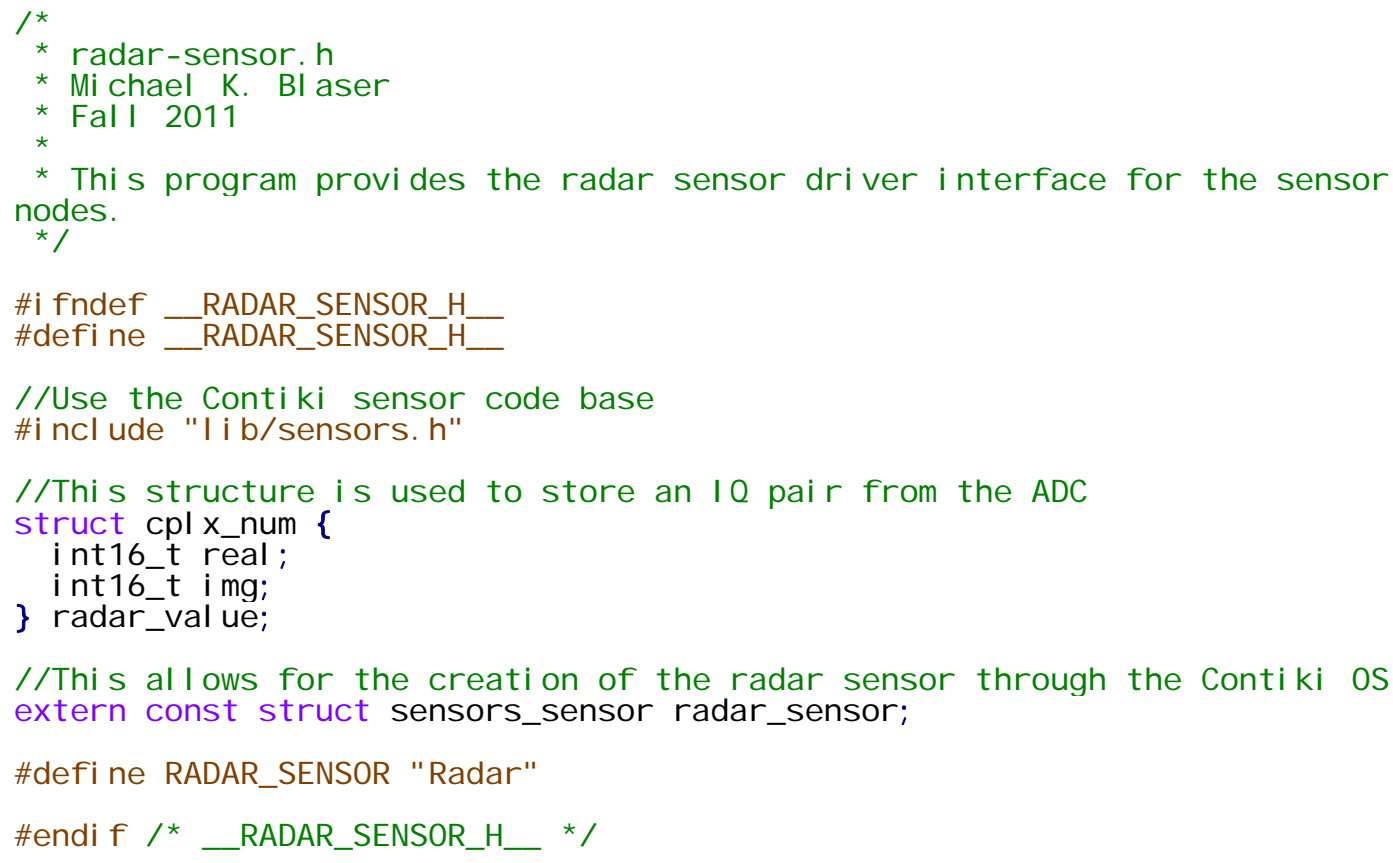




\section{radar-sensor.c}

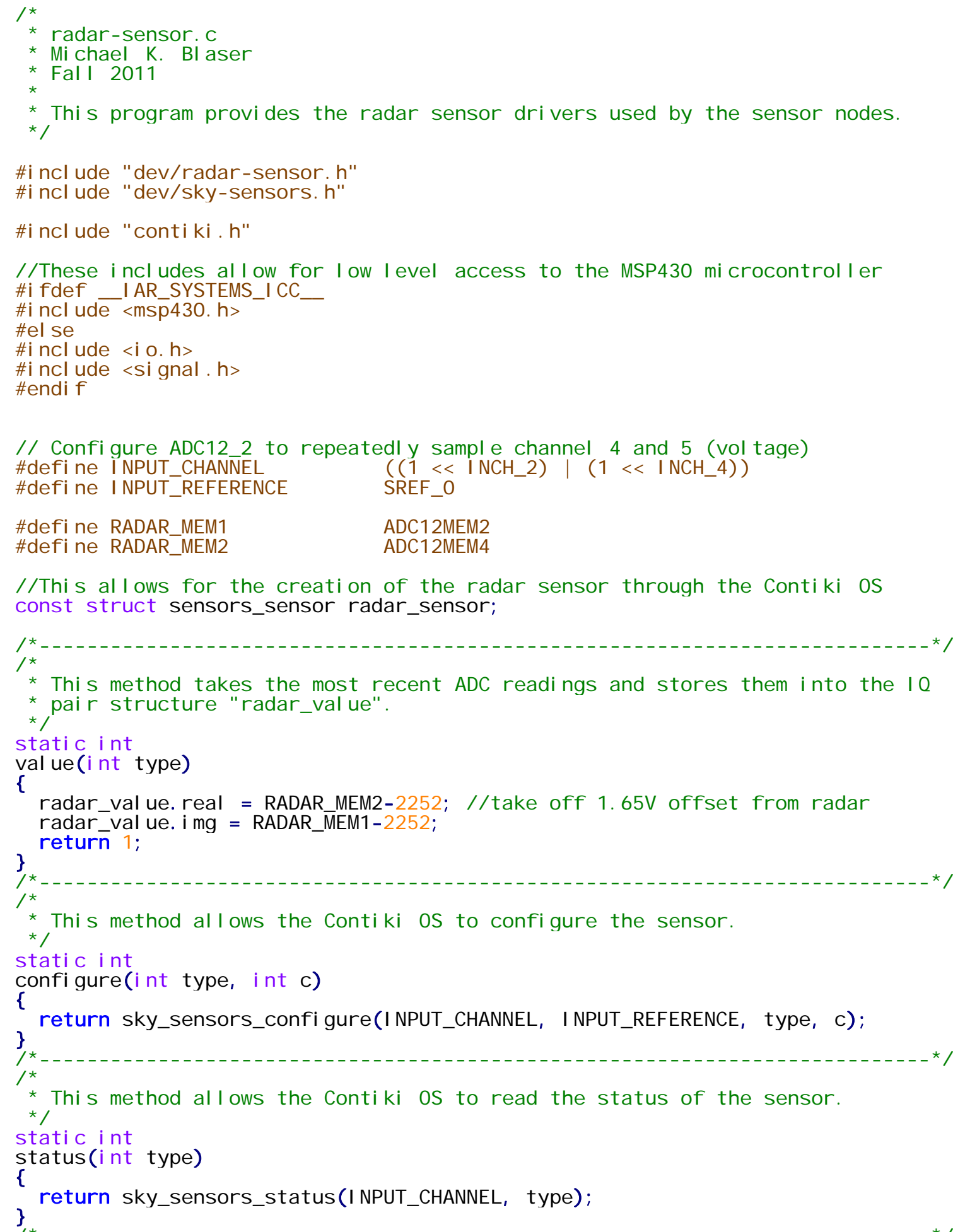


// This sets up how the sensor appears to the contiki os

SENSORS_SENSOR(radar_senSOr, RADAR_SENSOR, val ue, configure, status);

\section{tracker.m}

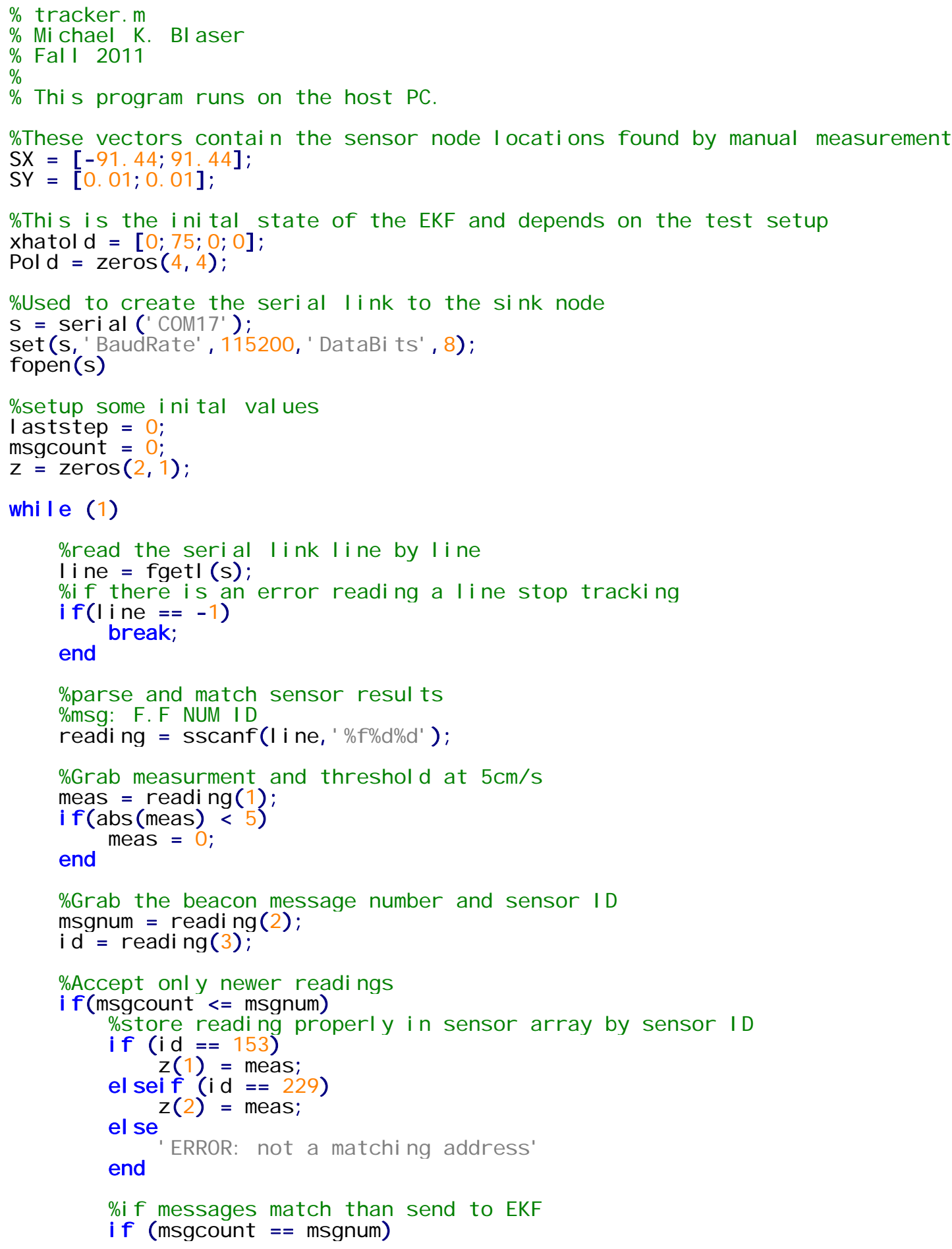




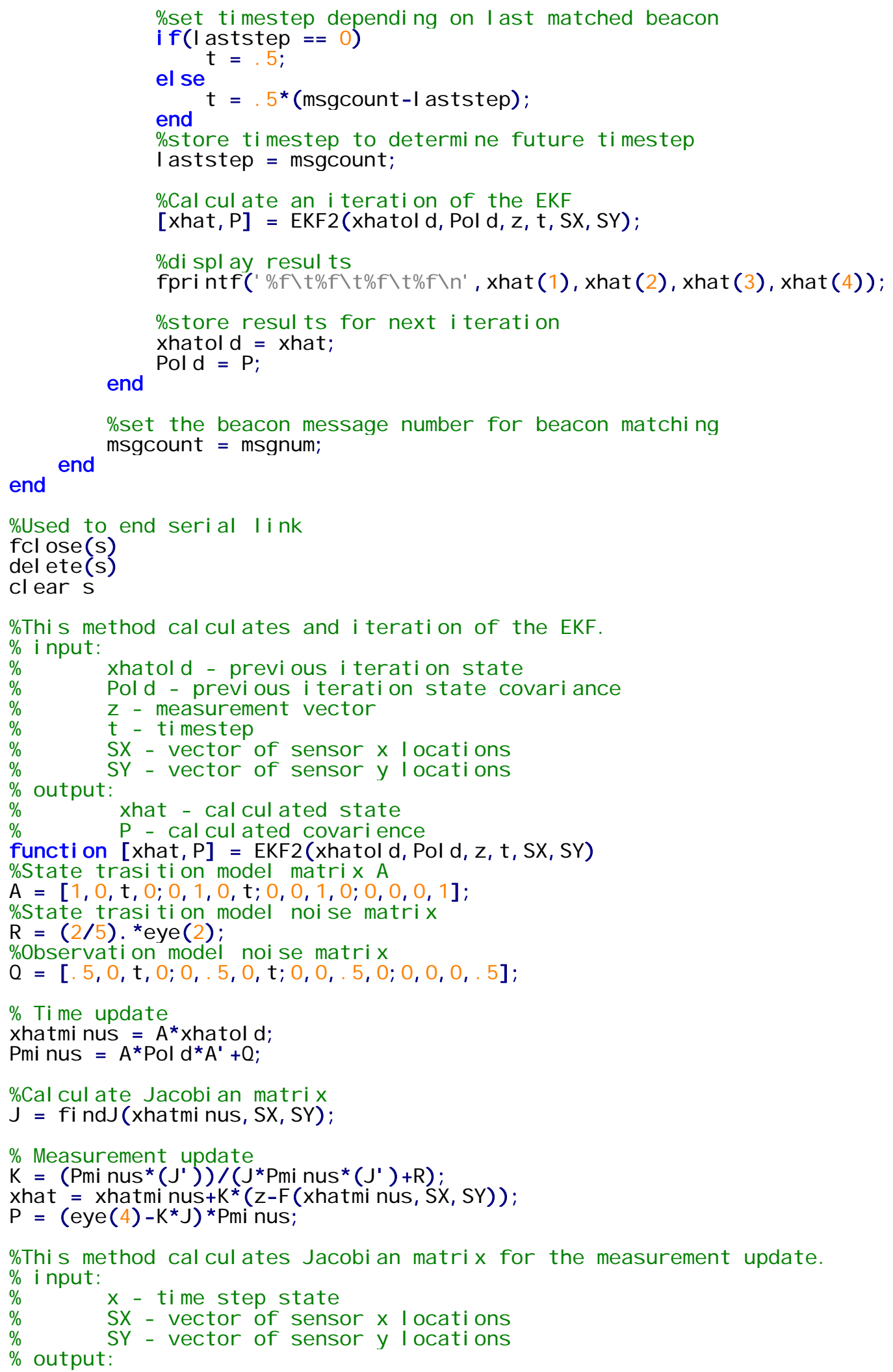




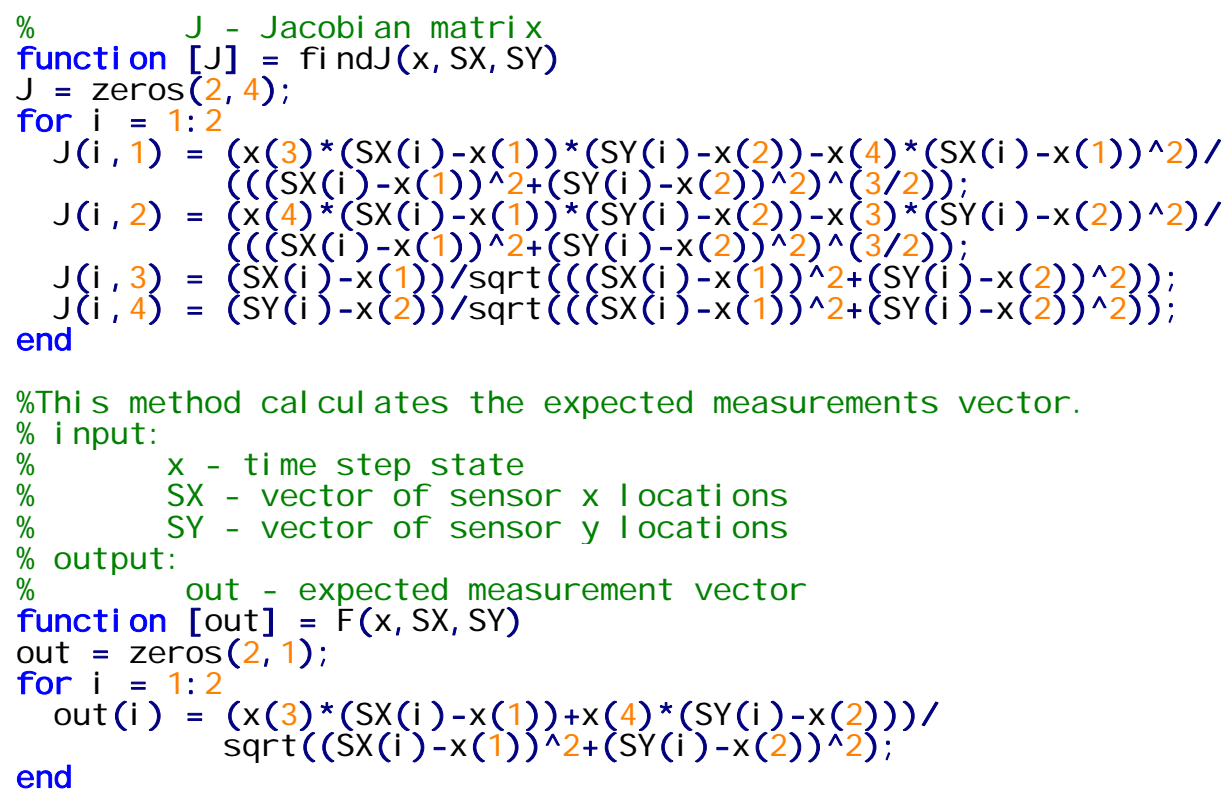




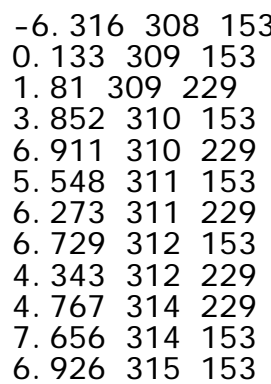

\section{Trial 3}

$5.502 \quad 315 \quad 229$

$6.379316 \quad 153$

11. $525 \quad 317 \quad 153$

4. $319316 \quad 229$

9. $310318 \quad 153$

8.308317229

9. $264318 \quad 229$

7. $316 \quad 319153$

$8.328 \quad 319229$

$6.416 \quad 320 \quad 153$

6.691321153

$9.578 \quad 320 \quad 229$
$8.953 \quad 322 \quad 153$

2. $763 \quad 321229$

1. $361322 \quad 229$

$3.206 \quad 323 \quad 153$

$3.808 \quad 323 \quad 229$

2. $598 \quad 324 \quad 153$

$3.900 \quad 324 \quad 229$

2. 599325229

$8.312325 \quad 153$

$11.1326 \quad 153$

$3.16326 \quad 229$

$8.115 \quad 327 \quad 153$
$0.29327 \quad 229$

1. 102328153

0.29328229

$0.0 \quad 329 \quad 229$

1. 821329153

$5.757 \quad 330 \quad 153$

$0.749330 \quad 229$

0.563331229

4. $106 \quad 331153$

0.210332229

$-1.101332153$

0.432333229

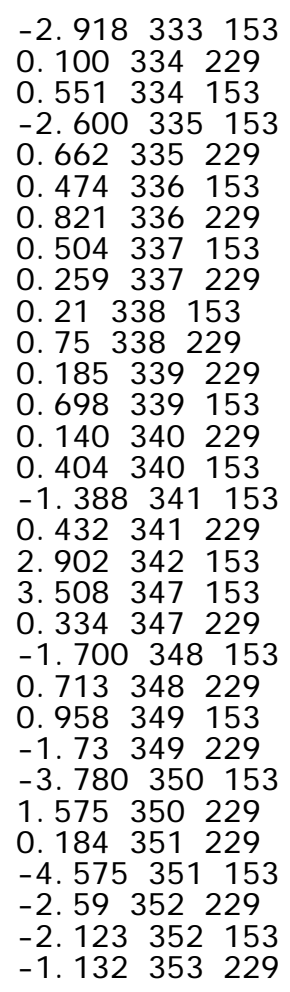

Trial 4
$-1.4353153$

- 1.674354153

- 4.140354229

$-5.836 \quad 355153$

0.507355229

$-6.827356153$

- 2.287356229

$-8.261357229$

- 6.807357153

$-1.27358153$

$-6.996358229$

.6 .610359229

$-1.754359153$

- 4. 138360153

$-3.551360 \quad 229$

- 4.732361229

$-4.911361153$

$-2.671362229$

$-8.576 \quad 362153$

$-3.334363229$

- 8.405363153

$-4.580364229$

- 4. 124364153

$-4.531365153$

- 3.189365229

- 2.924366229

$-5.152366153$

$-9.398367153$

$-7.34367229$

- 9.216368153

$-3.746368229$
$-5.764 \quad 370 \quad 153$

$-1.86 \quad 370 \quad 229$

$-4.753371153$

$-2.663371229$

$-7.191372153$

$-2.457372229$

$-7.481373153$

$-4.693373229$

- 3.213374153

- 3.496374229

$-5.72 \quad 375153$

$1.689376 \quad 153$

0.299375229

7. 213377153

$0.746 \quad 376 \quad 229$

7. 913377229

3.847378229

$8.566378 \quad 153$

$10.759379 \quad 153$

5. 877379229

8.642380229

12. $111380 \quad 153$

8. 226381153

11. $298 \quad 381229$

$6.344 \quad 382 \quad 153$

5.83382229

$7.85383 \quad 153$

6. 231383229

5. $598 \quad 384229$

9. $276384 \quad 153$

9. $305385 \quad 153$
$3.419 \quad 385 \quad 229$

$6.121386 \quad 153$

5. $106 \quad 386 \quad 229$

$10.296 \quad 387229$

7.71387153

$8.734388 \quad 153$

2. 213388229

6. $108 \quad 389 \quad 153$

0.542389229

$0.146 \quad 390 \quad 229$

$4.59390 \quad 153$

5. 37391153

0.133391229

0.0392229

$10.100 \quad 392 \quad 153$

7. $946 \quad 393 \quad 153$

0.0393229

3. $343 \quad 394 \quad 153$

$0.376 \quad 394 \quad 229$

2. $203 \quad 395 \quad 153$

$0.345 \quad 395 \quad 229$

0.131396229

0.680396153

0.148397229

6. 433397153

5. $428 \quad 398 \quad 153$

$0.960398 \quad 229$

$.4 .75399 \quad 153$

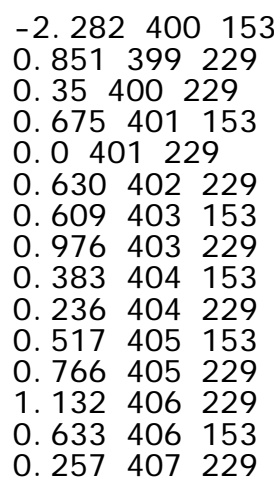

$0.420 \quad 407 \quad 153$

0.502408229

0.207408153

$0.880 \quad 414 \quad 153$

0.492414229

- 1.671415153

0.521415229

$-4.160416153$

2. $994 \quad 416 \quad 229$

1. 705417153

- 1.955417229

0.691418229

1. 989418153

$0.743 \quad 419 \quad 229$

$-2.291419153$
$-3.710 \quad 420 \quad 153$

$-1.187420 \quad 229$
-8.2864210

$-8.286421 \quad 153$

- 2.49421229

- 3.401422229

- 4.995422153

- 6.109423229

- 2. 136423153

0.152424153

$0.744 \quad 424 \quad 229$

$-6.252425153$

$-2.85425229$

- 5.490426153

.6 .63427153

$-1.76426229$
$-6.54428153$

- 4.415427229

$-6.707428229$

$-2.993429153$

- 2. 281430153

$-2.867429229$

- 5. 196431153

$-5.549430 \quad 229$

- 2. 994431229

$-7.934432153$

- 6.152433153

$-6.81432229$

$-5.782433229$

$-3.472434153$

- 1.365434229

B-2 
$-6.454436153$

$-3.773436229$

- 8.86437153

$-5.226437229$

$-10.991438153$

$-1.656438229$

$-2.726 \quad 439229$

$-5.901439153$

$-3.936440 \quad 229$

- 2. 177440153

$-6.408441153$

$-3.904441229$

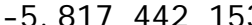

- $3.340 \quad 442 \quad 229$

$-5.233444153$

\section{Trial 5}

$\begin{array}{llll}-1.496 & 467 & 153 \\ 0.743 & 467 & 229 \\ 0.620 & 468 & 153 \\ 0.363 & 468 & 229 \\ -2.71 & 469 & 153 \\ 0.633 & 469 & 229 \\ 0.160 & 470 & 229 \\ 0.537 & 470 & 153 \\ -1.398 & 471 & 153 \\ 0.0471 & 229 \\ 1.157 & 472 & 153 \\ 0.547 & 472 & 229 \\ 0.587 & 473 & 229 \\ 0.391 & 473 & 153 \\ 0.754 & 474 & 229 \\ 1.173 & 474 & 153 \\ 0.843 & 475 & 229 \\ 0.929 & 475 & 153 \\ -1.461 & 478 & 229 \\ 1.150 & 479 & 153 \\ -1.247 & 480 & 153 \\ -3.371 & 479 & 229 \\ 0.328 & 480 & 229 \\ 0.597 & 482 & 153 \\ 0.262 & 482 & 229 \\ -8.438 & 483 & 153 \\ 0.24 & 483 & 229 \\ -4.321 & 484 & 153 \\ -2.345 & 484 & 229 \\ -1.340 & 485 & 153\end{array}$

Oblique

\section{Trial 1}

1. 177531153

$-1.225532153$

0.691531229

0.132532229

0.651533153

$0.270 \quad 533229$

$-6.110534153$

$0.584 \quad 534 \quad 229$

$-4.576535153$

$0.695 \quad 535229$

0.965536153

$0.96536 \quad 229$
$-2.66 \quad 443 \quad 229$
$4.613 \quad 444 \quad 229$
7. $855 \quad 445 \quad 153$
5. $304 \quad 445 \quad 229$
$10.652 \quad 446 \quad 153$
$5.813446 \quad 229$
9. $380 \quad 448 \quad 153$
6. $917448 \quad 229$
9. $485 \quad 449229$
$9.979449 \quad 153$
$9.822 \quad 450 \quad 153$
$0.207450 \quad 229$
5.513451153
$0.746 \quad 451 \quad 229$
$5.736 \quad 452 \quad 153$

5. $224 \quad 452 \quad 229$

$5.427 \quad 453 \quad 229$

$13.787 \quad 453 \quad 153$

4.82454153

$0.840 \quad 454229$

4. $334 \quad 455 \quad 153$

4. $994 \quad 455 \quad 229$

2. $574 \quad 456 \quad 153$

8. $227 \quad 456 \quad 229$

2. $883457 \quad 153$

2. $903457 \quad 229$

7. $597458 \quad 153$

2.645458229

5. $794 \quad 459 \quad 153$

0.212459229
$0.906 \quad 460 \quad 229$

0.292460153

1. 767461153

3. $948 \quad 461229$

5. $377462 \quad 153$

$0.715462 \quad 229$

7. $80 \quad 463 \quad 153$

$0.774 \quad 463229$

0.756464229

3. 953464153

$0.315465 \quad 153$

0.793465229

$0.278 \quad 466 \quad 153$

0.0466229
$-3.543501229$

- 6.235502153

- 4.989502229

$-7.306503153$

$-4.828503229$

$-4.542504153$

- 2.859504229

- 6.811505229

$-6.41505153$

0.731506229

$-2.759506 \quad 153$

3. $521507 \quad 153$

2. 2507229

$10.72 \quad 508 \quad 153$

$8.995 \quad 508 \quad 229$

4. 0509153

8. 218509229

9. $136 \quad 510 \quad 153$

8.713510229

$11.956511 \quad 153$

4.94511229

$6.246512 \quad 153$

4. $800512 \quad 229$

5. $15 \quad 513153$

4. $304513 \quad 229$

4.365514153

4. $403 \quad 514 \quad 229$

7. 971515229

$5.65 \quad 516229$
$9.845516 \quad 153$
8. $134517 \quad 153$

$5.889517 \quad 229$

$11.58 \quad 518 \quad 229$

5. 605518153

6. 93519153

3. 944519229

7. $50 \quad 520 \quad 153$

3. $330 \quad 520 \quad 229$

5. 561521153

0.379521229

1. $144522 \quad 229$

$0.744523 \quad 153$

1.65523229

3. 628524153

2. $325 \quad 524 \quad 229$

7. 532525153

0.755525229

0.0526229

4. 643526153

2. $811527 \quad 153$

0.69527229

0.365528229

0.864528153

0.322529229

2. 304529153

5. 504530153

- $1.234 \quad 530 \quad 229$

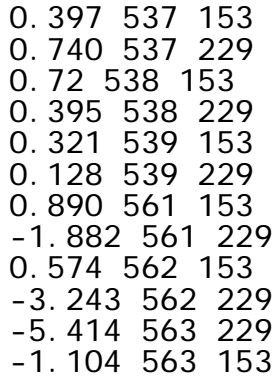

$-4.89571153$

- 1. 314571229

$-6.738572153$

- 4.993572229

- 9.112573153

- 2.254573229

0.947574229

- $1.684574 \quad 153$

- 2. 114575229

$-5.880 \quad 575153$

- 3.97576229

$-4.468576153$

B-3 


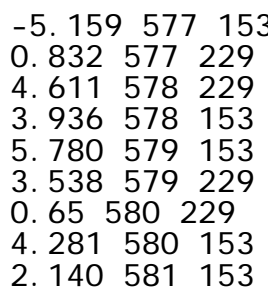

Trial 2
6.332581229

$4.239582 \quad 153$

$5.738582 \quad 229$

$7.122583 \quad 153$

$2.541583 \quad 229$

2. $818 \quad 585 \quad 153$

1. 235585229

$0.342586 \quad 153$

5. $273 \quad 586 \quad 229$

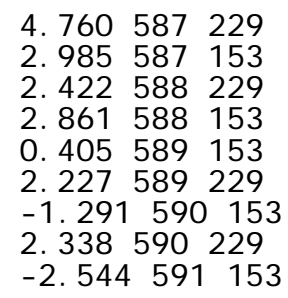

$1.248 \quad 591229$

1. 193592153

0.230593153

5. $881592 \quad 229$

1.821593229

- 1.391594153

1. 124594229

- 1.884595153

0.11595229
$0.684596 \quad 153$
0.0596229
0.708597229
0.510597153
0.251598229
1. 79598153
0.119599229
$-1.104599153$
$0.138 \quad 600 \quad 153$
0.257600229

$$
\begin{aligned}
& -1.204601153 \\
& 0.623601229 \\
& 0.990 \quad 602 \quad 153 \\
& -1.536602229 \\
& 0.840603 \quad 153 \\
& \text { 1. } 195603229 \\
& 0.898 \quad 604 \quad 153 \\
& 0.687 \quad 604 \quad 229 \\
& 0.750607229 \\
& 2.63607 \quad 153
\end{aligned}
$$

$$
\begin{aligned}
& -5.755 \quad 608 \quad 229 \\
& \text { - } 1.201608153 \\
& -1.172610229 \\
& 0.637610153 \\
& 0.142611229 \\
& -2.402611153 \\
& \text { - } 4.915612229 \\
& 0.219612153 \\
& 0.822613229 \\
& 0.700 \quad 613 \quad 153
\end{aligned}
$$

$0.507 \quad 614 \quad 229$

0.981614153

3. $596 \quad 615 \quad 153$

0.872615229

$-2.72616229$

$0.155 \quad 616153$

$-5.887617229$

$-4.476 \quad 617153$

\section{Trial 3}

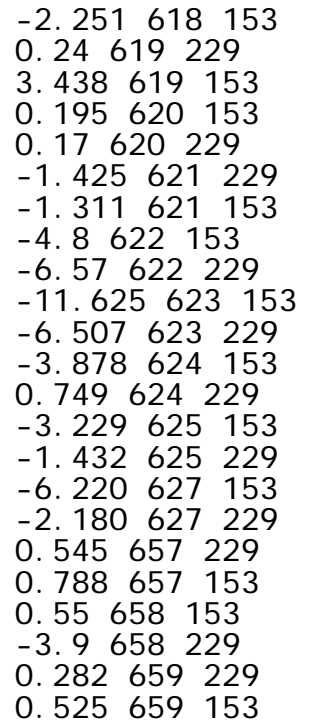

- 2. 251618153

3.43861915

$0.195 \quad 620 \quad 153$

0.17620229

- 1.311 621153

$-4.8622153$

- 11.625623153

$-6.507 \quad 623 \quad 229$

$-3.878 \quad 624 \quad 153$

- 3.229625153

$-1.432625229$

- 2.180 $627 \quad 229$

$0.55658 \quad 153$

0.282659229

$0.525659 \quad 153$

\section{Trial 4}

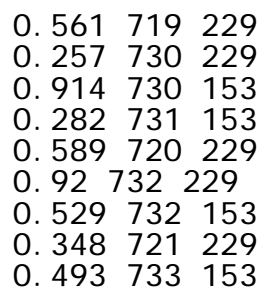

$$
\begin{aligned}
& 2.58 \quad 660 \quad 153 \\
& \text { - } 6.160660229 \\
& 0.19661229 \\
& \text { 3. } 216 \quad 661153 \\
& \text { 2. } 813 \quad 662 \quad 153 \\
& 0.871662229 \\
& \text { 1. } 618663153 \\
& -1.825663229 \\
& \text { 1. } 492664153 \\
& -2.680664229 \\
& \text { - } 3.365665153 \\
& 0.630665229 \\
& -2.258666229 \\
& \text { - } 2.245666153 \\
& -2.596667229 \\
& -2.774667153 \\
& 0.855 \quad 668 \quad 153 \\
& 0.716 \quad 668229 \\
& -5.213669229 \\
& 0.657669153 \\
& -3.801670 \quad 229 \\
& -3.433670 \quad 153 \\
& .9 .617671153
\end{aligned}
$$
$1.415 \quad 684 \quad 153$
2. 149685229
1. 940685153
1. 514686229
1. $90686 \quad 153$
$8.135 \quad 688 \quad 229$
0.752688153
$0.388 \quad 689 \quad 153$
5. 467689229
$0.938 \quad 690229$
1. 106690153
4.992691229
0.632691153
4.813692229
$-4.274 \quad 692 \quad 153$
1.755693229
$-2.90693153$
1.735694229
$0.230 \quad 694 \quad 153$
2. 419695153
4. $14 \quad 695 \quad 229$

4. $926 \quad 683229$

1. 312684229

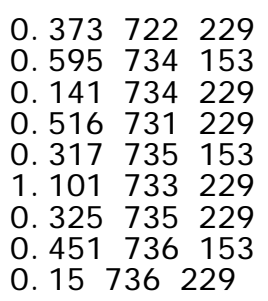

$$
\begin{aligned}
& -3.856 \quad 740 \quad 153 \\
& 0.623740229 \\
& -1.931741153 \\
& \text { - } 2.422741229 \\
& 0.906742 \quad 153 \\
& 0.351742229 \\
& \text { 2. } 111743153 \\
& \text { 1. } 192743229 \\
& 0.19744153
\end{aligned}
$$

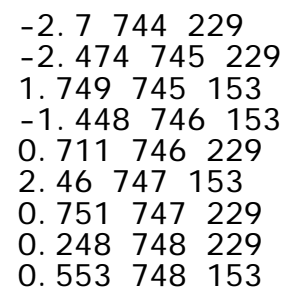




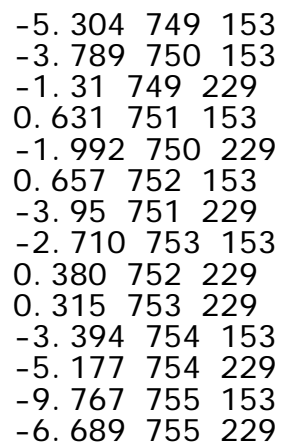

\section{Trial 5}

- 4. 1776153

1. 294777229

0.390777153

0.686778153

$0.85778 \quad 229$

1. 562779153

$-1.23779229$

1. $519780 \quad 229$

0.684780153

0.602781153

0.26781229

1. 192782229

0.182782153

$0.834783 \quad 153$

0.121783229

0.359785153

0.55785229

1. 137786153

0.842786229

$-2.353787229$

$0.808787 \quad 153$

$-3.864788229$
$-5.556756 \quad 153$

$-1.420756229$

- 6.479757153

- 2.196 758153

0.391757229

- 2. 429758229

- 1.689759153

0.224759229

$2.752 \quad 760 \quad 153$

$6.671760 \quad 229$

2. 646761153

5.622761229

$5.698 \quad 762 \quad 229$

10.164763153
5. $839 \quad 763 \quad 229$

2. 171764153

3. $206764 \quad 229$

0.742765153

0.464765229

0.52766153

2. $291766 \quad 229$

8. 434767229

0.25767153

5. 46768153

$6.916 \quad 768 \quad 229$

$6.575 \quad 769 \quad 229$

$1.796 \quad 769 \quad 153$

$-2.42770153$
$6.741770 \quad 229$

5.464771229

$-2.847771153$

- 1.407772153

1. 460772229

$0.945773 \quad 153$

3. $177 \quad 773 \quad 229$

0.327774153

6.269774229

- 2.285775153

3.666775229

$0.878 \quad 776 \quad 229$

\section{Diamond}

\section{Trial 1}

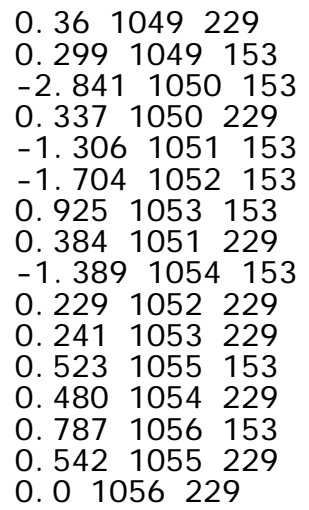

0.509800153

$-4.346801229$

$-6.542801153$

$-5.264802153$

$-2.781802229$

$-3.865803229$

$-5.989803153$

- 1.23804153

- 6.393804229

$0.814805 \quad 153$

0.332805229

$-5.947806153$

- 6.705806229

$-3.94807153$

0.392807229

7.690808153

7. $458 \quad 808 \quad 229$

3. 491809229

3. 139809153

6. $350 \quad 810 \quad 229$

2. $547810 \quad 153$

6. 43811229
0.211811153

5. $324812 \quad 229$

2. 251812153

3. 99813229

4. 15813153

$-1.3814153$

2. 84814229

$0.498 \quad 815153$

9. 227815229

6. 965816229

0.903816153

2. 681817229

3. 65817153

0.311818153

5. 92818229

$-4.559819153$

- 2. 222820153

4.542819229

0.238821153

4. 519820229

3. $288 \quad 822 \quad 153$

5. 506821229

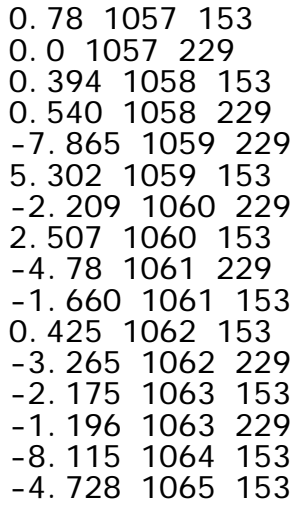

- 8. 1411064229

- 4. 3121066153

$-1.974 \quad 1065229$

$0.116 \quad 1067153$

$-6.430 \quad 1066 \quad 229$

$-1.1081068153$

1.491067229

- 11.2491069153

- 3.9521068229

$-7.941069229$

- 9.8431070153

0.01070229

2. 3031071153

7. 1601071229

9.8271072229

4.5031072153
$0.996 \quad 1073 \quad 153$

10.4331073229

1. 9541074153

6. 4871074229

2. $308 \quad 1075 \quad 153$

$1.688 \quad 1075229$

$10.652 \quad 1076 \quad 153$

8.4811076229

$14.478 \quad 1077 \quad 153$

8.187 1077 229

9.5081078153

0.2601078229

0.01079229

$18.460 \quad 1079 \quad 153$

0.01080229

5.5371080153

B-5 


\section{Trial 2}

\section{$-2.594 \quad 864 \quad 153$
0.74}

0.741864229

$-1.643865153$

0.774865229

0.28868153

0.187868229

0.457869153

$0.257870 \quad 229$

0.957870153

0.137871153

0.279871229

$-1.95872153$

0.663872229

0.607873153

0.154873229

$$
\begin{aligned}
& -1.190 \quad 874 \quad 153 \\
& 0.440 \quad 875 \quad 153 \\
& 0.192876153 \\
& 0.111891229 \\
& 0.295892153 \\
& \text { 4. } 59892229 \\
& \text { 5. } 821893153 \\
& -1.368893229 \\
& \text { - } 4.424894229 \\
& 0.247894 \quad 153 \\
& -1.665895153 \\
& \text { - 5. } 447895229 \\
& -4.375896153 \\
& 0.905897153 \\
& -5.522896229
\end{aligned}
$$

.6 .100898153

$-1.623897229$

$-9.500 \quad 899153$

$-5.66898229$

- 3.902900153

0.632899229

$-6.756901153$

$-4.189900 \quad 229$

$-7.130901229$

$-5.344903229$

$-10.597903153$

5. 29904153

$16.36 \quad 904 \quad 229$

2.792905229

4.781905153
$1.918906 \quad 153$

8. $111906 \quad 229$

3.874907153

6. $287907 \quad 229$

5. 969908229

$3.440 \quad 909229$

5. 460908153

$8.196 \quad 909 \quad 153$

7. 56910229

0.447911229

14. $238910 \quad 153$

16. 392911153

0.113912229

0.226913229

\section{Trial 3}

1. $393923 \quad 153$

0.09921229

2. $474924 \quad 153$

0.169923229

2. $757 \quad 925 \quad 153$

-2.806 924229

$-2.138925229$

- 2. 237926229

$-2.886926 \quad 153$

- 2.265927153

- 4.757927229

- 3.152929229

- 1.930929153

$-9.969930153$

.7 .94930229
$-7.42931153$

$0.516 \quad 954 \quad 153$

$0.866 \quad 954 \quad 229$

$3.847 \quad 955 \quad 153$

$0.850 \quad 955229$

0.392956229

7. 95956153

1. $689957 \quad 153$

0.522957229

$-2.848958 \quad 153$

0.513958229

$-1.312959153$

- 3.962960153

- 2. 52959229

- 4.109960229

- 3.349961229
.6 .177961153

0.837962229

$-6.323962153$

$-7.856963153$

$0.751 \quad 963229$

0.186964229

$-4.849964153$

- 2. $240 \quad 965153$

0.635965229

$-15.324966153$

2. 191966229

$-4.277967153$

6.972967229

2. $754968 \quad 153$

9.721968229

$14.385970 \quad 229$
$0.376 \quad 970 \quad 153$

15. 128971229

$-1.189971153$

5. $282972 \quad 153$

$9.723 \quad 972 \quad 229$

9.576 $973 \quad 153$

4.773973229

$15.215 \quad 974 \quad 153$

0.802974229

5. $394 \quad 975 \quad 153$

0.753975229

$7.656 \quad 976 \quad 153$

0.0976229

3. 625977153

\section{Trial 4}

0.717977229

$-1.38978153$

$0.379 \quad 978 \quad 229$

$.1 .748 \quad 979 \quad 153$

0.348979229

- 1.141980153

$0.0 \quad 980229$

0.939981229

$-2.567981153$

$0.747 \quad 982 \quad 153$

$0.266 \quad 982 \quad 229$

$0.740 \quad 983229$

$0.550 \quad 983 \quad 153$

$0.50 \quad 984 \quad 229$

$0.298 \quad 984 \quad 153$
$0.588 \quad 985 \quad 153$

$0.116 \quad 985 \quad 229$

0.158986153

0.626993153

0.136992229

1. 844994153

0.799993229

-2. 121994229

2. $418995 \quad 153$

$0.359995 \quad 229$

$1.835996 \quad 153$

$-1.802996229$

0.0999153

$-5.962999229$

. 1. 4551000153
$-4.999 \quad 1000229$

$-4.7511001153$

$-3.8261001229$

$-3.4651003153$

$-4.6491003229$

.4 .6651004153

- 8. 1591004229

- 11.31005153

0.6321005229

$-7.1951006153$

1.9841006229

13. $107 \quad 1007229$

3.8891007153

$11.670 \quad 1008 \quad 229$

1.6021008153
13. $35 \quad 1009 \quad 229$ 4.9981009153

2. 6471010153 4.9511010229

8.7091011153 2. 6291011229

12. 9011012153 11. 4221012229 15. $214 \quad 1013 \quad 153$ 7. $539 \quad 1013229$ 7.3201014153 $-1.7861014229$ 13. $95 \quad 1015153$

0.01015229

\section{Trial 5}

4. $474 \quad 1016153$

2. $6131017 \quad 153$

0.1051016229
0.1031017229

$-1.31018153$

0.6281018229 $\begin{array}{lll}0.208 & 1019 & 153 \\ 0.625 & 1020 & 153 \\ 0.671 & 1019 & 229\end{array}$

0.7231021153

0.2031022153
0.521020229

B-6 
Appendix B Datasets

$\begin{array}{lllll}0.621021229 & -3.3021031 & 153 & -8.9201038153 \\ -1.2411023153 & -6.1461030229 & -4.4091031229 \\ 0.1431022229 & -4.2731032153 & 1.2131039153 \\ 0.8031023229 & -10.6281033153 & -1.8211032229 \\ 0.1041025229 & -7.6131034153 & 4.4621040153 \\ 0.6511026229 & 1.1271034229 & 4.411041153 \\ 6.1321027153 & -6.2721035153 & -1.6261033229 \\ -1.7541027229 & 1.9651035229 & 2.4011042153 \\ 2.6171028153 & -1.9211036153 & 3.6011039229 \\ 0.8991028229 & -11.6641036229 & 14.6231040229 \\ -2.5741029153 & -11.9201037153 & 8.31041229 \\ -3.4921029229 & -4.111 .037229 & 1.2201043153 \\ 0.821030153 & 8.6421038229 & 3.991042229\end{array}$

$0.26 \quad 1043 \quad 229$

$8.596 \quad 1044153$

5.742 $1044 \quad 229$

$13.138 \quad 1045 \quad 153$

1.6531045229

$8.3811046 \quad 153$

$3.904 \quad 1046229$

20.6431047153

0.01047229

6.91 1048153

0.1241048229

$-3.492102922$

$-4.1111037229$

1. $2201043 \quad 153$ 